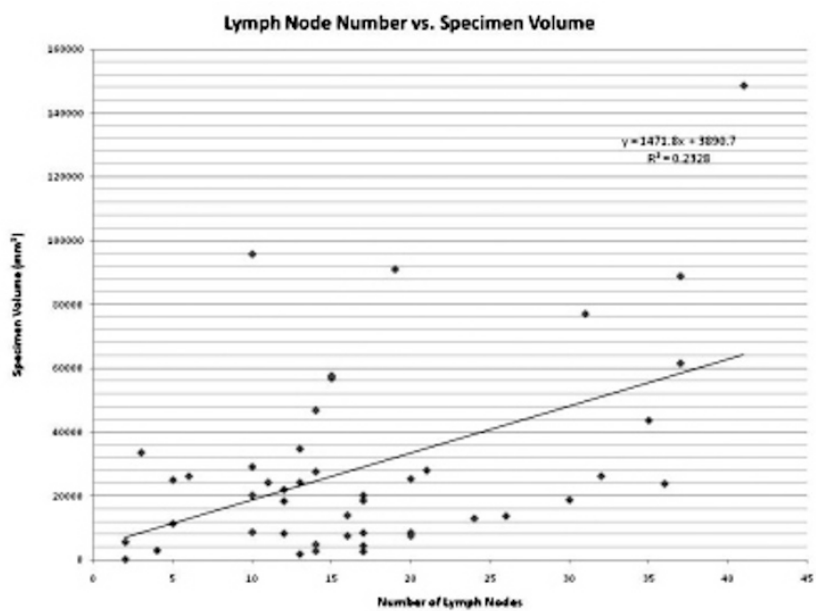

LN positivity did not correlate with either $\mathrm{LN}$ number $(\mathrm{p}=.28)$ or volume $(\mathrm{p}=.57)$. Conclusions: The number of LN identified in LA is variable and does not correlate with lymphoid tissue volume. Variability in LN numbers may result from counting sectioned single LN as multiple LN. A standard method of dissecting pelvic LN should be developed before LN numbers are used in treatment protocols or as study eligibility criteria.

1870 Pre-Dissection Review of Ultrasound Report Increases Accuracy of Gross Thyroid Tumor Measurements.

LYA Watts, LA Emery, A de las Morenas. Boston Medical Center, MA.

Background: Cancer staging is invariably dependent on primary tumor size. However, discrepancies may exist between preoperative and postoperative measurements, which may affect staging. There are systematic ways in which organs are evaluated according to predetermined dissection techniques. In the thyroid, serial slices are made perpendicular to the long axis of each lobe. Measurements of lesions found are impacted by these initial incisions and may engender the discrepancy from radiographic measurements. We propose a pre-dissection evaluation of the ultrasound (US) report so that the initial cut is made into the lesion in the plane of maximum dimension.

Design: Retrospective patients were screened from our pathology database by searching total thyroidectomy specimens. Patients included in the analysis had discrete nodules measured on gross pathology as well as on US. Prospective patients were those receiving total thyroidectomies with US reports showing dominant nodules. Thyroids flagged as having dominant nodules were grossed with particular attention to the plane of maximum dimension, in that the initial cut into the lesion was made in this plane. Thus, the correlation between gross and US measurements for retrospective patients was made after gross dissection whereas for prospective patients the US measurements were used as a guide for dissection. For both retrospective and prospective patients, gross pathology measurements were compared to US measurements of the dominant nodule(s) and differences in maximum dimension were recorded.

Results: Six retrospective and six prospective patients were analyzed. The retrospective group was found to have a mean maximum difference between US and gross measurements of $0.67 \mathrm{~cm}$ (SD:0.4) and the prospective group $0.1 \mathrm{~cm}$ (SD: 0.06). Results were statistically significant with a 2-tailed p-value of 0.011 (CI: 0.161-0.972). Conclusions: Predissection review of the US report in a patient with a dominant thyroid nodule guides pathologic evaluation to more accurately measure tumor size, which ultimately may impact patient management.

1871 Application of Lean Principles To Improve Turn Around Time in Gynecologic Cytology Laboratory.

RJ Wong, M Harigopal, K Schofield, D Chhieng, A Levi. Yale University School of Medicine, New Haven, CT.

Background: Our cytology laboratory, like many others, is under pressure to improve quality and provide test results faster while decreasing costs. We sought to address these issues by applying Lean principles and methodologies. The objective is to determine the impact of Lean principles on the turn around time (TAT) and productivity of the gynecologic cytology operation.

Design: We established a baseline measure of the TAT of Pap tests. We then compared this to performance 6 month after implementing the location guided imaging system and Lean methodologies. The latter included workstation and equipment relocation, redesign of workflow, and visual control.

Results: The average TAT for Pap tests before and after the implementation of new imaging system and Lean principles were $5.98 \pm 0.93$ days and $2.60 \pm 0.38$ days, respectively. The improvement in the average TAT was statistically significant (t-test $p$ $<0.001)$. In addition, there was also significant reduction in the variability as evidenced by the reduction in the standard deviation (F-test, $\mathrm{p}<0.001$ ).

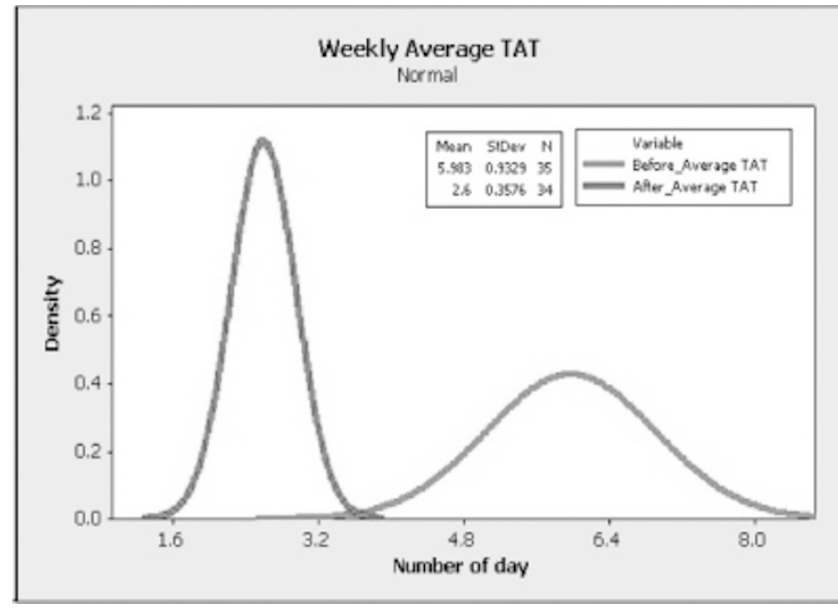

In addition, the productivity of staff was improved by $17 \%$, i.e. the same volume of Pap tests were handled by 2 fewer full-time cytotechnologists who voluntarily resigned. Conclusions: Implementation of Lean principles in cytology laboratory resulted in shortened and more consistent TAT for Pap tests while improving productivity at the same time.

\section{Pan-genomic/Pan-proteomic Approaches to Diseases}

1872 High Levels of Necrosis in TCGA Glioblastoma Samples Are Associated with the Mesenchymal Gene Expression Class and Characterized by Enhanced Expression of Master Transcriptional Regulators of Mesenchymal Transition.

DJ Brat, DA Gutman, LAD Cooper, J Kong, C Chisolm, EG Van Meir, JH Saltz, CS Moreno. Emory University School of Medicine, Atlanta, GA.

Background: The Cancer Genome Atlas (TCGA) project defined four clinically relevant subsets of glioblastoma (GBM) based on gene expression: mesenchymal, classical, proneural and neural. Genomic alterations were identified for each class, yet complete correlation with expression was not evident, suggesting tumor micro-environment may contribute. We investigated if the degree of necrosis within GBM samples used for TCGA studies was associated with a specific expression class and examined which genes were most tightly correlated with extent of necrosis.

Design: We utilitzed publicly available digitized frozen section slides from 79 GBMs used for quality assurance before molecular testing by TCGA. Necrotic foci in each slide were outlined using a machine-human interface. Extent of necrosis (\% tissue area) was correlated with expression class. Using the Significance Analysis of Microarray (SAM) survival module, a Cox regression analysis was performed using TCGA Affymetrix U133 expression data to identify genes correlated with \%-necrosis. The SAM survival module used a permutation algorithm to correct for multiple hypothesis testing and identify correlated probes with a false discovery rate cutoff of $<4 \% .1026$ probes were positively associated with \%-necrosis and 18 were negatively associated. These 1044 probes were used as input for Ingenuity Pathway Analysis (IPA) to identify enriched networks.

Results: The mesenchymal expression class was enriched with high \%-necrosis GBMs ( $33 \%$ with $>25 \%$ necrosis) compared to the other 3 classes ( $2 \%$ with $>25 \%$ necrosis) Average \%-necrosis was higher in mesenchymal GBMs (21.2\%) than the other 3 classes $(7.4 \%$; one-way ANOVA $\mathrm{p}=0.0022)$. Regression analysis of genes correlated with $\%$-necrosis revealed strong upregulation of transcription factors identified as master regulators of mesenchymal transition (Carro, et al. Nature 263:318-25, 2010), including C/EBP-B, C/EBP-D, FOSL2, STAT3 $(\mathrm{p}<0.005)$ and RUNX1 $(\mathrm{p}<0.01)$. Increased expression of hypoxia-inducible genes, including VEGF, validated the analysis. FOSL2 and C/EBP-B were strongly correlated with extent of necrosis within the mesenchymal class $(\mathrm{p}<0.00001)$.

Conclusions: Our results suggest that the degree of necrosis in GBM is closely related to the mesenchymal gene expression class.

1873 Application of Reverse Phase Protein Lysate Array (RPPA) to Formalin-Fixed, Paraffin-Embedded Tissues.

R Broaddus, S-S Xie, C Wei, E Euscher. M.D. Anderson Cancer Center, Houston, TX. Background: RPPA is a high-throughput analysis in which dilutions of protein lysate are spotted on nitrocellulose slides (maximum 1,152 spots per slide). Each slide is probed with a different antibody. A DAKO-catalyzed signal amplification system is used for signal detection. RPPA requires less protein than Western blots. An advantage over immunohistochemistry is that RPPA is quantitative rather than qualitative. A current limitation of RPPA is that protein is typically derived from cell lines or frozen tissues, restricting its use to research settings. The RPPA platform incorporates many antibodies directed to phosphorylated proteins; these same antibodies typically do not work well for immunohistochemistry using formalin-fixed, paraffin-embedded (FFPE) tissues. It would therefore be advantageous if RPPA could be adapted to FFPE tissues. 
Design: We examined FFPE uterine carcinosarcoma (CS; $n=52)$. Uterine CS is a rare but highly malignant type of endometrial cancer with limited availability of frozen tissue. Fifty micrograms of protein were extracted from FFPE uterine CS, one paraffin block per case. Integrity of the protein was verified by Western. RPPA was performed using a panel of 225 antibodies directed towards proteins and phosphorylated proteins important in cancer cell growth regulation. ANOVA was used to calculate differences in protein expression between different subgroups of uterine CS.

Results: Protein differences were noted in the uterine CS cases with heterologous sarcomatous differentiation (presence of rhabomyosarcoma or chondrosarcoma). Twenty-eight proteins were differentially expressed in CS with heterologous components, including nine phosphorylated proteins (pARAF, pSRC, pSTAT3, pATM pER, pX4EBP1, pYB1, pFAK, and pNFKB). Several of the differentially expressed proteins, EGFR, ER, HER2, VEGFR2, AKT, and SRC, are key components of cell signaling pathways that can be targeted by drugs currently in clinical trials.

Conclusions: The application of RPPA is feasible using FFPE tissues. RPPA therefore has the potential to be used in the clinical laboratory for routine cancer patient care. Using a combination of traditional H\&E based light microscopic examination and RPPA, we have shown that uterine CS with heterologous sarcomatous elements differentially express transcription factors, DNA repair proteins, and cell growth regulatory proteins. These proteins may be important for targeted therapy of uterine CS as well as deciphering the molecular pathogenesis of these clinically aggressive neoplasms.

1874 Mass Spectrometry-Based Glycoproteomic Profiling Identifies Distinct Protein Biomarkers of Hodgkin Lymphoma.

NA Brown, CM McNeil, V Basrur, F Damian, KP Conlon, DS Rolland, A van den Berg, $S$ Poppema, KSJ Elenitoba-Johnson, MS Lim. University of Michigan, Ann Arbor; University of Groningen, Netherlands.

Background: Hodgkin lymphoma is one of the most frequent lymphomas with $95 \%$ representing Classical Hodgkin Lymphoma (CHL) and 5\% Nodular Lymphocyte Predominant Hodgkin Lymphoma (NLPHL). While the tumor cells of both entities derive from germinal center B cells, these two lymphomas are clinically and genetically distinct. Overlapping histologic and immunophenotypic features of these lymphomas make this diagnostic distinction challenging in some cases. Since a majority of N-linked glycoproteins are either localized on the membrane or secreted, these proteins are attractive targets for the identification of biomarkers that could aid in the distinction of these two lymphomas.

Design: Using an innovative proteomic strategy targeting N-linked glycosylated proteins, we characterized the glycoproteomic profiles of CHL and NLPHL. Cell lines derived from NLPHL (DEV) and CHL (L428, L1236 and KMH2) were used for solid-phase extraction of N-linked glycopeptides which were identified and quantified using liquid chromatography and tandem mass spectrometry. A subset of proteins was selected for validation using Western blot analyses or flow cytometry.

Results: Glycopeptides from over 250 proteins were identified from each of the four cell lines using false discovery rate of $<4.5 \%$. This included over 90 cluster designated (CD) proteins. Over $90 \%$ of the peptides contained the NXST motif which identifies distinct sites of asparagine glycosylation. Importantly, mass spectrometry data correlated strongly with known proteins expressed by CHL (CD30 and CD15) and NLPHL (CD45, CD19, CD20, CD22, CD79A, CD79B, and IgH). In addition, many novel glycoproteins (adhesion molecules, cytokine receptors and immune regulatory molecules) that were selectively expressed by CHL and NLPHL were identified. Orthogonal analyses by Western blotting and/or flow cytometry corroborated the results obtained by MS/MS with expression of three markers selectively identified in NLPHL and one in CHL.

Conclusions: We identified glycoproteins that had not been previously associated with either CHL or NLPHL and may serve as diagnostic markers. Orthogonal approaches corroborated the results obtained by mass spectrometry. This study demonstrates that mass spectrometry-based glycoproteomic profiling can be used for discovery of novel biomarkers and phenotypic profiling without antibodies.

1875 Testing and Validating High Throughput Genomic Analysis of FFPE Samples: Systematic Gene Expression Differences between Matched Frozen and FFPE Samples Due to Rapid mRNA Degradation Signals.

$R$ Cabal, $R$ Khanin, A Viale, $N$ Socci, $R$ Lucito, A Olshen, J Zhang, W Gerald, $S$ Powers, M Ladanyi. Memorial Sloan Kettering Cancer Center, New York, NY; Cold Spring Harbor Laboratory, Cold Spring Harbor, NY; University of California, San Francisco.

Background: To assess the performance of current platforms in comprehensive genomic profiling of formalin-fixed paraffin-embedded (FFPE) samples, we used duplicate frozen and matched FFPE tumor samples of 5 lung adenocarcinomas (total=20 samples) to evaluate array-based and next generation sequencing-based DNA and RNA profiling approaches.

Design: For genomic copy number profiling, we compared Affymetrix SNP 6.0 arrays, Affymetrix MIP arrays, Agilent $1 \mathrm{M}$ arrays, and ABI SOLiD low coverage sequencing. For transcriptional profiling, we evaluated Illumina whole genome DASL arrays, Affymetrix Exon arrays, and ABI SOLiD transcriptome sequencing, using Affymetrix U133Plus 2.0 array data from the frozen tumors as a reference.

Results: Agilent $1 \mathrm{M}$ arrays and $\mathrm{ABI}$ SOLiD low coverage sequencing performed best by different measures including noise, replicate concordance, and matched frozenFFPE concordance. ABI SOLiD sequencing showed better reproducibility of frozen vs FFPE replicates than any of the array platforms. ABI SOLiD sequencing (even low coverage) outperformed various array platforms in both transcriptional and genomic copy number profiling on FFPE samples. Correlations between expression data from frozen samples and FFPE samples were worse than any cross-platform correlations using the same sample type, suggesting that there are systematic gene expression differences between matched frozen and FFPE samples. We performed an unbiased search of 3'UTRs for 7-9 bp motifs that found an AU-rich motif (AAAUAUU) to be significantly associated $(r=0.73)$ with gene expression differences between FFPE and frozen. Similarly, the average number of the canonical AUUUA mRNA degradation signals showed a significant linear relationship $(r=0.78)$ to fold-changes between FFPE and frozen

Conclusions: ABI SOLiD sequencing outperforms various array platforms in both transcriptional and copy number profiling on FFPE samples. AU-rich motifs are significantly over-represented in mRNAs that are downregulated in FFPE vs frozen samples, suggesting a possible relationship to AU-rich rapid mRNA degradation signals. This result suggests a biological, rather than technical, basis for the discordance between expression profiles of FFPE vs frozen samples.

\section{TLR4 and MyD88 Expression in Cancer Stem Cells.}

AA Cooke, MF Gallagher, SA O'Toole, CM Martin, OM Sheils, JJ O'Leary. University of Dublin, Trinity College, College Green, Ireland.

Background: Ovarian cancer is the most common cause of gynaecologic cancer death in the western world. Although most patients respond positively to initial therapy, a high proportion of patients will face the development of chemoreistant recurrent disease over their lifetime. One suggested mechanism for this is the cancer stem cell theory. Cancer stem cells (CSCs) are a minority population of cancer cells which display stem like characteristics including enhanced proliferation. They are also known to display chemoresistance.

Design: Cell line experiments were carried out in two CSC lines: NTera2 and 2102Ep. NTera2 cells are pluripotent and readily differentiate in response to retinoic acid treatment while 2102Ep cells are nullipotent and resist differentiation via retinoic acid. Cells were incubated in the presence of both retinoic acid and cisplatin. RNA was isolated from treated cells and interrogated for mRNA expression via Q-PCR.

Results: Both NTera2 and 2102Ep cells showed alterations in the TLR4/MyD88 pathway following treatment with retinoic acid. Following on from this it was decided to treat both cell lines with cisplatin and assay for a possible TLR4 response. Both cell lines showed an alteration in TLR4 signalling, however in opposite directions. Given the difference in chemosensitivity between the two cell lines we hypothesise that this difference is important in chemoreistance of these cells. Experiments involving predifferentiation of cells via retinoic acid followed by cisplatin treatment were also performed. We are currently performing functional analysis on the TLR4 pathway to clarify its potential role in chemoresistance.

Conclusions: Our results show for the first time that TLR4 and MyD88 are involved in the cellular response to both differentiation and cisplatin treatment. The difference in response between the two cells when viewed with the differing chemosensitivies of the two lines suggest that this difference may be involved in their levels of chemoresistance. The TLR4/MyD88 mechanism was also found to be linked with differentiation, chemoresistance and differentiation avoidance. We are currently performing further experiments to investigate this. Acknowledgments Funded by Cancer Research Ireland

1877 The Epithelial Mesenchymal Transition (EMT) Profile of Platelet Cloaked Cancer Cells.

DJ Crowley, PA Smyth, SA O'Toole, K Egan, D Kenny, OM Sheils, JJ O'Leary. Trinity College Dublin, Ireland; Royal College of Surgeons Ireland, Dublin, Ireland.

Background: Ovarian cancer (OC) has a mortality rate of $75 \%$ and is the leading cause of death from gynaecological malignancy. Almost half of patients diagnosed with OC present with thrombocytosis. This may be associated with Tumour CellInduced Platelet Aggregation (TCIPA), observed to protect platelet 'cloaked' cancer cells from TNF- $\alpha$-, natural killer cell- and chemotherapeutic agent-mediated death, and enhance their capacity for metastasis. Epithelial-Mesenchymal Transition (EMT) is a programme of cell development employed during embryonic development, and also in oncogenesis and metastasis where it is characterised by loss of cell adhesion, increased mobility, invasiveness and chemoresistance. The present study was to determine if TCIPA mediates its enhancement of ovarian cancer cells' capacity to metastasise through induction of EMT.

Design: The effect of platelet releasate concentrations on cell lines representing normal ovarian epithelium (HIO-80) and ovarian epithelial cancer (59M, SK-OV 3, A2780, A2780cis) was optimised by MTT assay. Cells were exposed to platelet releasate (PLR) or equivalent concentration of washed platelets (WPL) for $8 \mathrm{~h}$. Total RNA was extracted using Qiagen RNeasy Kit and cDNA was prepared for use in TaqMan assay of key markers of EMT: ALDH1A1, AKT1, CDH1, PIK3CA, SNAIL1, SNAIL2, TWIST1, VIM, ZEB1 and ZEB2. Expression changes were determined using the Spotfire Software suite.

Results: TaqMan analysis of EMT markers in ovarian cell lines exposed to washed platelets or platelet releasate indicates significant $(\mathrm{p}<0.05)$ alteration of some genes: - HIO-80 cells showed increased SLUG expression in response to WPL exposure. $\bullet 59 \mathrm{M}$ cells showed increased CDH1 and reduced AKT1 expression in response to both WPL and PLR, reduced SNAIL expression and increased ZEB1 expression in response to WPL and PLR exposure respectively. A2780cis cells increased VIM expression in response to both conditions and increased expression of TWIST in response to PLR.

Conclusions: These data indicate a differential response to platelet releasate and washed platelets in cell lines reflecting epithelial health from normal to malignant ovarian disease. Thus, in platelet cloaked metastasis, while cloaking may not be sufficient to independently drive EMT, it may contribute to establishing or maintaining a pro-EMT signal in metastasising ovarian cancer cells. This work is supported the Irish Health Research Board (HRB) PhD Programme in Molecular Medicine: From Genes to Function. 
1878 Platelet Cloaking of Cancer Cells Alters Apoptotic Potential: Implications for Metastasis.

DJ Crowley, PA Smyth, CD Spillane, SA O'Toole, K Egan, D Kenny, OM Sheils, JJ O'Leary. Trinity College Dublin, Ireland; Royal College of Surgeons in Ireland, Dublin, Ireland.

Background: Ovarian cancer (OC) is the leading cause of death from gynaecological malignancy. OC frequently presents with thrombocytosis, possibly associated with Tumour Cell Induced Platelet Aggregation (TCIPA). TCIPA has been observed to protect platelet 'cloaked' cancer cells from TNF- $\alpha-$, NK cell- and chemotherapeutic agent-mediated destruction, and enhance their metastatic capacity. This study aims to determine the genetic basis for this enhancement though whole transcriptome analysis of cells exposed to washed platelets (WPL) or platelet releasate (PLR)

Design: PLR concentrations for use on cell lines representing normal ovarian epithelium (HIO-80) and ovarian epithelial cancer (59M, SK-OV 3, A2780, A2780cis) were optimised by MTT assay. Cells were exposed to PLR or equivalent WPL for $8 \mathrm{~h}$. Total RNA was extracted, prepared for Affymetrix Human Exon Array and analysis was performed using Affymetrix Gene Chip QC and Biotique Systems XRAY software. Twenty six significantly altered genes were validated by Fluidigm Dynamic Array for Gene Expression

Results: XRAY analysis indicates context-specific significant changes $(\mathrm{P}<0.05$; fold change $>1.5$ ) in gene expression in response to PLR and WPL exposure. Ovarian cancer cell lines 59M and SK-OV 3 returned the greatest response to PLR exposure with A2780cis, HIO-80 and 59M responding most to WPL. These exposures altered expression of genes involved in; $\$$ 99: anti-apoptotic, anti-autophagy, pro-angiogenic and proliferative signalling and reduced expression of genes important in maintaining genomic stability. $\bullet$ SK-OV 3: cell cycle, metabolic and transcriptional regulation, regulators of TSGs/oncogenes. A2780cis: anti-apoptosis, transcriptional regulation, protease expression and cell-cell interaction. $\bullet \mathrm{HIO}-80$ : metabolic and transcription regulation

Conclusions: Relative changes in gene expression in exposure groups compared to control group indicate a context-defined response in a panel of ovarian cell lines to either PLR or WPL. We hypothesise that these changes indicate TCIPA-induced pro-metastatic signalling, mediating its effects through mechanical platelet-cancer cell signalling and via the content of platelet granules released during this interaction. This results in pro-angiogenic, proliferative and anti-apoptotic signalling in addition to regulation of translation, cell cycle and metabolism to enhance successful metastasis. This work is supported the Irish Health Research Board (HRB) PhD Programme in Molecular Medicine: From Genes to Function.

1879 Detection of Prognostically Significant AML Translocations and Single Base Mutations by Next Generation Sequencing.

EJ Duncavage, HJ Abel, S South, JD Pfeifer. University of Utah, Salt Lake City; Washington University, St Louis, MO.

Background: Acute Myeloid Leukemia (AML) is classified by the presence of recurrent cytogenetic abnormalities. Current methods for detection of these abnormalities offer relatively low sensitivity, and are often unable to define cryptic or uncommon translocation partners. Correlation with molecular studies for mutations in specific genes is also recommended, adding to testing complexity. We developed a targeted next generation sequencing (NGS)-based approach for clinical AML samples to identify both single gene mutations and translocations, including those with unique partners.

Design: Biotinylated RNA capture probes were designed to tile across introns and exons of genes implicated in AML risk assessment and comprised a target area of $1 \mathrm{Mb}$. Patient genomic DNA, extracted from bone marrow of AML patients with known recurrent cytogenetic abnormalities, was captured and sequenced on an Illumina GAII using 60bp paired-end reads. Using DNA barcodes we sequenced 4 samples in multiplex on a single GAII lane. The resulting sequence data were then aligned to the targeted gene regions and translocations/large scale structural variations identified by the SLOPE software package.

Results: We previously sequenced one AML case with a known $\mathrm{t}(9: 11)$ translocation in a single GAII lane, resulting in 1,100x coverage and successful identification of the translocation. In contrast, when the assay was multiplexed with 4 samples the average coverage became highly variable, ranging from $900 \mathrm{x}$ to $20 \mathrm{x}$. We identified putative translocations in only the two cases with the greatest coverage, while mutations in single genes such as FLT3, NPM1, and CEBPA were identifiable in all cases.

Conclusions: Single and multiplex targeted NGS can be used to identify translocations and prognostically significant single gene mutations in AML. While translocations are easily detected in singly sequenced specimens, the current results demonstrate that the cost savings of multiplexed sequencing are offset by decreased and variable coverage, resulting in an inability to identify translocations in some cases. The data indicate, however, that longer read lengths will overcome these problems.

1880 Novel Stat3 Targeted Mouse Model of Colitis Mimics Tumor Progression in Human Ulcerative Colitis.

KFriedman, EY Lin, J Albanese, E Liu, K Tanaka, A Leifer, Q Liu. Montefiore Medical Center of Albert Einstein College of Medicine, New York, NY.

Background: To determine the link between inflammation and tumorigenesis in intestine, we have recently established a novel mouse model of colitis-induced colorectal cancer (Stat3-IKO mice), by targeting Stat 3 specifically in hematopoietic cells including macrophages and certain populations of lymphocytes. Inflammation was initiated in the colon of the mutant mice at early age that was followed by the development of malignancies including invasive adenocarcinoma in the inflamed region of the intestine. We have previously demonstrated that this model shares several characteristics with human ulcerative colitis (UC)-associated colorectal cancers including the involvement of intestinal microflora, activation of oncogenic pathways in colonic epithelial cells and disrupted epithelial homeostasis.

Design: To characterize tumor progression in Stat3-IKO mice, tissue was collected in 60 Stat3-IKO and control mice at 5, 10-14, 26-27, and greater than 30 wks of ages to assess for inflammation, low and high grade dysplasia, and invasive adenocarcinoma. Furthermore, immunostaining was used to identify the potential tumor-promoting factors produced in the inflammatory microenvironment in both human UC and mouse colon.

Results: We found that inflammation in the colon was initiated at early age ( $5 \mathrm{wks}$ ) in Stat3-IKO mice. At 10-14 wks, low grade dysplasia was present in $60 \%$ of the Stat 3 IKO mice. Older Stat3-IKO mice (26-27 wks) showed more severe inflammation and the lesion had progressed to high grade dysplasia (44\%) and invasive carcinoma (22\%). Finally, all three Stat3-IKO mice examined at greater than 30 wks have developed invasive adenocarcinoma. As seen in human UC-associated carcinoma, pathways involved in inflammation-induced neoplastic transformation including Toll-like receptors (TLRs), chemokines and their receptors as well as S100A9 are found to be upregulated in the inflamed colon and tumor lesions in Stat3-IKO mice.

Conclusions: Tumor progression in Stat3-IKO mice shares many morphologic features with human UC-associated adenocarcinoma. The histopathological development in the model follows the sequence of inflammation, dysplasia and then carcinoma suggesting that inflammation plays a critical role in tumorigenesis, which is compatible with that observed in human UC. Our study demonstrated that Stat3-IKO mice is a valuable pre-clinical model for illustrating the mechanism of inflammation-induced neoplastic transformation in colon, and may be useful for the development of therapeutic strategy for human UC.

\section{FGFR2 Alterations in Endometrial Carcinoma.}

$S$ Gatius, A Azueta, A Velasco, M Santacana, J Pallares, X Matias-Guiu. Hospital Universitari Arnau de Vilanova, University of Lleida, IRBLLEIDA, Spain.

Background: Fibroblast growth factor receptor 2 (FGFR2) is a tyrosine kinase receptor involved in many biological processes such as embryogenesis, adult tissue homeostasis and cell proliferation. Mutations in FGFR2 have been reported in up to $16 \%$ of endometrial carcinomas (EC) identical to those found in congenital craniofacial disorders. Inhibition of FGFR2 could be a new therapeutic target in EC.

Design: FGFR2 immunostaining was assessed in three tissue microarrays (TMA): one constructed from paraffin-embedded blocks of 70 samples of normal endometrium (NE) in different phases of menstrual cycle, and two TMAs containing EC samples ( 95 and 62 cases). FGFR2 expression was correlated with stage, histological type and grade as well as with immunostaining of PTEN, RASSF1A, estrogen and progesterone receptors and KI67. FGFR2 mutations were assessed by PCR and direct sequencing, with DNA obtained from 31 paraffin-embedded EC samples and corresponding normal tissues. Results: In NE, FGFR2 expression was higher in the secretory than in the proliferative phase ( $\mathrm{p}=0,001)$. Cytoplasmic expression of FGFR2 was higher in EC when compared with $\mathrm{NE}(\mathrm{p}=0,0283)$, particularly when comparing NE with endometrioid carcinomas (EEC). Interestingly, nuclear staining was observed in some cases, and it was less frequent in $\mathrm{EC}$ when compared with $\mathrm{NE}(\mathrm{p}=0,0465)$. There were no statistical differences when comparing superficial and myoinvasive tumor samples. EEC showed higher expression of FGFR2 than non-endometrioid carcinomas, but the difference lacked statistical significance. Grade III EEC showed decreased FGFR2 expression when compared with grade II EEC $(p=0,009)$. No differences were found regarding pathological stage. Two missense mutations of FGFR2 gene were detected in exons 6 and 11 (S252W and N540K respectively), each of them in EEC (6.45\%). None of the remaining 29 cases exhibited any mutation.

Conclusions: FGFR2 cytoplasmic expression is higher in EC in comparison with NE, which support a role of FGFR2 as oncogene in EC, and suggests that FGFR2 could be a possible therapeutic target in EC. However, the frequency of mutated cases in this series seems to be lower than in other previous series.

1882 Gene Expression Profiling of Colonic Serrated Epithelial Lesions.

TS Giles, D Chiao, E Stelow, P Pramoonjago, CA Moskaluk. University of Virginia, Charlottesville.

Background: A subset of serrated epithelial lesions in the colon have been found to have an increased risk of malignant degeneration. These lesions have been termed sessile serrated adenomas or sessile serrated polyps (SSP) to distinguish them from hyperplastic polyps (HP) that have no or minimal risk of progression to carcinoma. Distinguishing SSPs from HPs may be problematic by histology, and finding distinguishing biomarkers may be of clinical utility.

Design: Histologic slides of serrated colonic epithelial lesions were reviewed and 10 consensus cases each of SSPs and HPs were selected. Corresponding tissue from the paraffin blocks was dissected, RNA was extracted and was screened for integrity using a quantitative RT-PCR analysis. Passing RNA samples were reverse transcribed, subjected to linear cDNA amplification and assayed on Affymetrix U133 Plus 2 oligonucleotide microarrays.

Results: 3 cases each of the SSP and HP cohort contained RNA of sufficient quality to be assayed by microarrays. The fluorescence intensity data was subjected to a metric that ranked genes on the basis of fold difference, absolute difference and statistical significance. The top 5 probes sets corresponding to annotated genes that distinguish SSPS from HPs are: MUC5AC, CYP2C18, PLA2G16, TMEM92 and BHMT2. The top 5 genes that distinguish HPs from SSPs are: MUC12, EXOC3, IGHA1, MS4A12 and SELENBP1. The table shows the probe designation, the gene name, the average fluorescence intensity for the SSP and HP samples, the fold change between SSP and HP samples and the P value ( 2 tailed t-test). 


Gene Expression Data
\begin{tabular}{|l|l|l|l|l|l|}
\hline Probe Set & Gene & SSP - Avg & HP - Avg & SSP/HP & P \\
\hline 214385 s at & MUC5AC & 1891 & 270 & 6.99 & 0.026 \\
\hline 208126 s at & CYP2C18 & 82 & 11 & 7.43 & 0.014 \\
\hline 235110 at & PLAG2G16 & 91 & 23 & 3.89 & 0.015 \\
\hline 235245 at & TMEM92 & 437 & 145 & 3.01 & 0.013 \\
\hline 242169 at & BHMT2 & 54 & 11 & 4.73 & 0.020 \\
\hline 1557906 at & MUC12 & 1039 & 4162 & 0.25 & 0.002 \\
\hline 228847 at & EXOC3 & 49 & 362 & 0.14 & 0.011 \\
\hline 215118 s at & IGHA1 & 75 & 407 & 0.19 & 0.006 \\
\hline 220834 at & MS4A12 & 68 & 954 & 0.07 & 0.035 \\
\hline 214433 s at & SELENBP1 & 149 & 520 & 0.29 & 0.001 \\
\hline
\end{tabular}

Conclusions: Our preliminary data suggest that there are patterns of gene expression that distinguish SSPs from HPs, and that representative gene expression profiles of these lesions can be obtained from a subset of archival formalin-fixed paraffinembedded samples.

\section{A Family of Novel miRNA-Like Small RNAs Dysregulated in Renal} Cell Carcinoma.

C Guo, X Wu, H Gao, B Mu, JM Jin, J Wang, M D'Apuzzo, LM Weiss, H Wu. City of Hope, Duarte, CA.

Background: Small non-coding RNA (smRNA) is involved in almost every biological process, including developmental timing, cell differentiation, cell proliferation, cell death, metabolic control, transposon silencing and antiviral defence. Some smRNAs are derived from tRNA, snoRNA and introns. MicroRNA (miRNA) is a group of smRNAs that regulate gene expression. It is processed from its precursors which act as substrates for Drosha and Dicer. Incorporating with Argonaute (Ago) proteins to form a miRNAinduced silencing complex (miRISC), miRNAs base-pair to target mRNAs and induce their translational repression or deadenylation and degradation. Recent study has shown several human snoRNAs with miRNA-like functions.

Design: (1) Deep sequencing of smRNA populations co-immunoprecipitated with Ago2 protein and Dicer from renal cell carcinoma $(\mathrm{RCC})$ cell lines $(\mathrm{n}=4)$; (2) functional characterization of selected smRNAs through a siRNA strategy; (3) whole genome smRNA and miRNA expression profiling by deep sequencing of a RCC frozen tissue cohort $(\mathrm{n}=12)$.

Results: (1) A population of smRNAs is bound to Ago2 protein and is associated with Dicer; (2) these smRNAs are composed of fragmented tRNA, rRNA, snoRNA, snRNA, scRNA, Mt-tRNA, introns and exons; (3) knock-down of several selected candidates from this smRNA family inhibits the proliferation of RCC cell lines; (4) in addition to known miRNAs, a number of these smRNAs are differentially expressed in RCC, as compared to benign renal tissue.

Conclusions: We have defined a novel family of smRNAs which are miRNA-like and are derived from other non-coding RNAs, introns and exons. This family of miRNAlike smRNAs is involved in regulation of RCC cell proliferation and is found to be aberrantly expressed in renal cell carcinoma, suggesting a functional role in RCC tumorigenesis.

\section{Altered microRNA Expression in Adenocarcinoma of Lung from} Smokers and Never-Smokers.

$M$ Guo, $M$ Huang, GA Calin, KR Hess, $W$ Zhang. The University of Texas M.D. Anderson Cancer Center, Houston; Fox Chase Cancer Center, Philadelphia, PA.

Background: Recent studies on the altered microRNA (miRNA) expression in lung cancer have shed light on our understanding in lung cancer carcinogenesis at epigenetic level. Identifying altered miRNA expression that is independent of tobacco smoking in lung cancer may enhance our understanding further on the epigenetic events in lung cancer that may lead to the development of new tests for early detection, prognosis and assessment for treatment response in lung cancer patients. In this study, we conducted miRNA profiling analysis using microarray assay in lung adenocarcinoma from smokers and non-smokers

Design: From the tumor bank at Fox Chase Cancer Center, we collected 50 lung adenocarcinomas with matched non-neoplastic lung tissue. In the cohort, 25 patients had no history of tobacco smoking. MiRNA expression profiling analysis was conducted using Human miRNA V2 Oligo Microarray Kit (Agilent Technologies, Alto, CA). Feature Extraction (9.5.3.1) software was used to link a feature to a design file and determine the relative fluorescence intensity of each sample. The validation tests were conducted using miRT-PCR assay (Applied Biosystems, Foster City, CA). The altered miRNA expression in lung adenocarcinoma was compared between smokers and non-smokers.

Results: In smokers, we identified 23 abnormally expressed miRNAs with 8 upregulated and 15 down-regulated. In non-smokers, 26 miRNAs were found abnormally expressed with 8 up-regulated and 18 down-regulated. MiR-21, mir-196a and miR$310 \mathrm{~b}$ were exclusively up-regulated in non-smokers, while miR-130a, miR-140-3p, miR-144a, miR-187* and miR-497 were exclusively down-regulated in smokers. Among the up-regulated miRNAs, mir-130b, mir-210, mir-503 and mir-21* were expressed in both smokers and never smokers. In down-regulated, miRNAs, miR-126*, miR-126, Mir-133b, miR-139-5p, miR-145*, miR-145, miR-218, miR-30*, miR-30a, miR-338-3p, miR-451, miR-468-5p and miR551b were expressed in both smokers and non-smokers. The altered expression of miR-310b, miR139-5p and miR486-5p were confirmed using real time RT-PCR.

Conclusions: The finding of miRNAs abnormally expressed in both smokers and non-smokers suggested that the altered miRNA expression that is independent of tobacco smoking may represent an epigenetic predisposition in carcinogenesis of lung adenocarcinoma

1885 Bimodally Expressed Genes in Ovarian Carcinoma.

AHS Hall, DN Kernagis, MB Datto. Duke University Medical Center, Durham, NC. Background: In certain cancers, genes can behave as molecular switches, having either an "on" or "off" state. These genes generally have what is statistically described as a bimodal distribution in expression. In the context of breast cancer, these switches are well described. They include ER and HER2 and define distinct cancer subtypes, which respond to different treatments and have different overall survival. Genes with a bimodal pattern of expression are also good targets for clinical testing because the difference in expression between "on" versus "off" populations is easy to measure. We hypothesized that bimodally expressed genes can be found in other cancers, specifically ovarian cancer, and that in this context they also define important biological tumor subtypes.

Design: We evaluated the largest publically available ovarian cancer dataset, which includes 245 malignant serous tumors, 18 serous tumors of low malignant potential and 20 malignant endometrioid tumors. We used a bimodal gene discovery algorithm to identify genes with a bimodal distribution. Correlations among expression of the bimodal genes, overall survival and tumor type were investigated using Fisher's exact test and standard Kaplan Meier survival analysis.

Results: We found many genes with prominent bimodal distributions of expression. Among the 174 genes with the strongest bimodal expression, we found ten genes that had a significant association with overall survival in malignant serous tumors, including placental alkaline phosphatase, JUB and interferon alpha 2. When expression of these genes was combined into a single survival index, the median survival for patients with malignant serous tumors with a favorable survival score was 65 months, versus 33 months for patients with malignant serous tumors with an unfavorable survival score $(\mathrm{p}<0.0001)$. We also found thirteen genes with significantly different expression between malignant serous tumors and endometrioid tumors and twenty-six genes with significantly different expression between malignant serous tumors and serous tumors of low malignant potential.

Conclusions: This work demonstrates that ovarian cancer, like breast cancer, can be classified by bimodal gene expression patterns. This approach provides insight into clinical behavior and identifies ideal targets for robust and precise clinical testing.

1886 Development and Analytical Validation of a Quantitative TissueBased Assay for Phospho-Smad2 (Ser465/467) in Glioblastoma Multiforme: A Potential Biomarker of TGF- Pathway Activation.

TR Holzer, M Gustavson, R Pinard, J Christiansen, L Graves, SP Myrand, SK Kadam MMF Lahn, JT Brandt, JM Yingling, A Nasir. Eli Lilly and Co., Indianapolis, IN; HistoRx, Branford, CT.

Background: Using immunohistochemical assays, we and others have shown that high p-Smad2 expression is associated with shorter survival in patients with glioblastoma multiforme (GBM). This study focuses on: 1) Development and optimization of a robust assay for quantification of p-Smad2 in archival GBM tissues from one institution and 2 Cross-platform validation of adverse prognostic significance of high nuclear p-Smad2 expression on an independent GBM cohort.

Design: The study group included 108 adult patients (Male:Female/68:34; Mean age: 53.7 years) with primary GBM treated with standard of care therapy at a single academic medical center. A multi-core GBM tissue-microarray (TMA) was stained for p-Smad2, using fluorescence immunohistochemistry (AQUA ${ }^{\circledR}$ technology). For each tumor, nuclear p-Smad2 (Ser465/467) expression was quantified as an AQUA score. Scores were compared among redundant cores from each case, using Pearson's correlation coefficient and GLM-ANOVA. An optimal AQUA score cut-point to differentiate cases with low and high p-Smad2 expression was determined with respect to 2-year overall disease-specific survival, using X-Tile ${ }^{\mathrm{TM}}$ graphical cut-point analysis program. KaplanMeier survival analysis and Cox Proportional Hazards modeling were performed using the low and high p-Smad2 expression groups of patients.

Results: Of the $108 \mathrm{GBM}$ cases, 89 were evaluable by AQUA analysis. AQUA scores for $\mathrm{p}$-Smad2 expression in redundant cores showed high similarity (Pearson's $\mathrm{R}=0.97$; GLM ANOVA P $=0.54$ ), suggesting good reproducibility. Using the optimal cut-point above, $62(70 \%)$ GBMs were categorized as low and $27(30 \%)$ GBMs as high p-Smad2 expressers. The high $\mathrm{p}$-Smad2 patient subset showed a significant $(\mathrm{p}=0.013)$ decrease in 2 -year disease-specific survival. The median survival of this group was 10 months vs. 13 months in patients with low p-Smad2 expression. Despite adjusting for age, a critical determinant for survival in GBM, high p-Smad2 expression remained significantly associated with decreased survival [HR $=1.92(95 \% \mathrm{CI}: 1.09-3.40)$; $\mathrm{p}=0.025]$.

Conclusions: Using a quantitative AQUA-based assay, high p-Smad2 expression was further substantiated as an adverse prognostic factor in primary GBM patients. Since $\mathrm{p}$-Smad2 is a member of the TGF- $\beta$ pathway, a quantitative assay may be clinically relevant for TGF- $\beta$ beta inhibitor clinical trials.

1887 Rapid and Reliable Method To Isolate Nuclei from FFPE Specimens for Genomic-Scale Molecular Profiling.

K Killian, L Wang, B Walker, A Glatfelter, WI Smith, PS Meltzer. National Cancer Institute, Bethesda, MD; Suburban Hospital, Bethesda, MD.

Background: Surgical pathology specimens are complex mixtures of benign and malignant cell types. Germ-line and lineage-related molecular signals arising from background benign cells may mask genomic and epigenomic aberrations present in malignant cells; this effect lowers the sensitivity of somatic mutation profiling. In contrast with FISH and PCR-type assays, which can make use of a few hundred cancercell nuclei/genome equivalents, high-resolution genome-scale profiling requires 3 orders of magnitude more input DNA. In addition, pathology specimens are formalin-fixed and paraffin-embedded, which may present additional challenges for large-scale cell and/or organelle fractionation. 
Design: This study was designed to develop a method for the large-scale, reliable and rapid purification of individual, unclumped, stable nuclei with minimal background debris from FFPE archival specimens. Details of specimen homogenization, lysis, washing, and storage were optimized to reduce processing time and improve yield using empirical measurements. Yield and purity of extracted nuclei were analyzed by DNA flow cytometry and florescence microscopy; performance in molecular profiling studies including high-resolution array comparative genomic hybridization was also tested. Technical replications were performed to test reliability of results.

Results: Details of specimen homogenization, lysis, washing, and storage were optimized to permit recovery of millions of FACS-ready nuclei from FFPE specimens in $<2$ hours. High-resolution array CGH assays manifest significant improvement in the efficiency of aberration detection.

Conclusions: We present a facile and rapid method for the extraction of copious amounts of nuclei from FFPE samples, and demonstrate excellent molecular profiling results with the nuclei fractions.

1888 Copy Number Variations among Renal Cell Neoplasms Exhibit Heterogenity Across Subtypes, Homogeneity within Subtypes and Correlated Changes in Transcriptional Profiles.

JM Krill-Burger, MA Lyons, CM Sciulli, LA Kelly, SI Bastacky, R Dhir, AV Parwani, WA LaFramboise. University of Pittsburgh, PA.

Background: DNA changes in a few gene loci have been identified in renal cell carcinomas (RCC), but the extent of familial and somatic DNA rearrangements underlying RCC is unknown. High density SNP arrays allow genome-wide interrogation of tumors at $1-2 \mathrm{~kb}$ resolution to characterize copy number variations $(\mathrm{CNV})$ and loss of heterozygosity ( $\mathrm{LOH}$ ). Application of molecular techniques to renal neoplasms may reveal DNA changes correlated with morphological differences and clinical behavior as well as provide new discriminants for tumors difficult to classify by current methods. Design: High-density SNP analysis (Affymetrix 6.0) and whole genome expression assays (Affymetrix exon arrays) were performed on DNA and RNA obtained from frozen renal tumors $(\mathrm{n}=18)$ plus "adjacent normal" renal tissue $(\mathrm{n}=5)$ and an in house reference library of normal tissue $(\mathrm{n}=17)$. Samples included papillary, oncocytoma, chromophobe and conventional specimens classified independently by 3 pathologists.

Results: Individual RCC tumors contained as few as 8 (papillary RCC) and as many as 4917 (chromophobe RCC) CNV segments. Adjacent normal renal tissue exhibited 10 to $97 \mathrm{CNV}$ changes compared to donor normal specimens. No significant differences in global CNV numbers and segment lengths were detected among tumor classes (mean+/-S.E.: 945+/-358) although they were consistently higher than adjacent normal tissue (39+/-16 CNV segments). No CNV region was common to all RCCs with highest overlap comprising a $26 \mathrm{~kb}$ segment at $15 \mathrm{q} 11.2$ found in $39 \%$ of RCC samples. However, common CNV segments were identified in individual RCC subtypes ranging from 2 amplifications present in all 4 oncocytomas to 212 amplifications found in 4 of 5 papillary RCCs. Whole genome expression studies demonstrated that significant changes in levels of mRNA and microRNA transcripts located within CNV segments correlated with copy number changes. Significant changes occurred in critical gene families associated with tumorigenesis including DNA repair (BRCA1, ATAD5, XRCC2), cell cycling (GRB2, ANLN, BOD1) and transcription regulation (FOXI1, RNF213).

Conclusions: These data reveal extensive global heterogeneity of $\mathrm{CNV}$ rearrangements in RCC including copy number variations in adjacent normal renal tissue. However, there was consistent, correlated overlap of $\mathrm{CNV}$ changes and encoded mRNA transcripts within individual RCC subtypes suggesting the presence of common diagnostic molecular signatures and distinct pathways that may serve as novel therapeutic targets for future studies.

1889 Comparing Two Different Platforms for Analyzing Genomic DNA Micro-Array in Mental Retardation.

A Leifer, Q Pan. Montefiore Medical Center, Bronx, NY

Background: Most recently, genomic DNA micro-array technology has revolutionized the field of cytogenetics that previously championed karyotyping and FISH. With expanding probe numbers, micro-array is even more effective at identifying any type of segmental imbalance, of any size, on the entire genome. This study serves as a way to evaluate product performance of two commonly used platforms.

Design: Complete genomes of 40 patient's were first assessed using Agilent 4x44k arrays. Of the 40 patients, 16 were affected individuals with major copy number changes, 16 were affected individuals with no major abnormalities, and 8 were normal, unaffected parents. The same patients were then reexamined by using Illumina Human CytoSNP 12 bead chip array and software.

Results: In the 16 patients with major aberrations, Illumina did not confirm two phenotypically critical duplications that were found by Agilent. In the 16 patients without major aberrations and the 8 normal patients, Illumina and Agilent did not find any significant copy number changes. In addition, Illumina recognized 16 normal CNV regions that were not found previously by Agilent. Furthermore, Illumina alone identified 10 UPD regions in the same data set, 6 from phenotypically affected individuals and 4 from normal individuals, with a maximum size of $8.5 \mathrm{Mb}$.

Conclusions: The data was analyzed with Agilent and then repeated with Illumina. Of the 16 patients with major aberrations found by Agilent, Illumina missed two significant copy number changes. Additionally, Illumina, with a higher density of probes in the array, found more normal CNVs. As expected, Illumina, which utilizes SNP probes detected UPD regions, which were of unknown clinical significance. However, large UPD regions in our cohort were not identified.
1890 Increased Expression of MicroRNA-210 in Microsatellite Instability High Endometrial Carcinoma and Its Potential Role in DNA Damage Repair.

D Ma, ZChen, H Cheng, B Djordjevic, KP Patel, MG Luthra, BA Barkoh, S-S Xie, SMa, $G$ Chen, J Medeiros, $R$ Broaddus, $R$ Luthra. The University of Texas M. D. Anderson Cancer Center Center, Houston.

Background: Endometrial carcinoma (EC) is the most common malignant neoplasm of the female reproductive tract. Young women $(<40$ years) with EC usually have a better prognosis and are managed conservatively. Approximately $20 \%$ of ECs are associated with microsatellite instability (MSI-high). MicoRNAs (miR) are small noncoding RNAs that regulate gene expression. Differential expression of miRs has been shown in MSI colorectal carcinomas. In this study, we report for the first time the miR expression profile in MSI-high and microsatellite stable (MSS) ECs.

Design: RNA was extracted from frozen endometrial tumors (15 MSI and 15 MSS) using the RNeasy Mini kit (Qiagen, Valenica, CA). 100 ng RNA was labeled with Cyanine 3-pCp and hybridized onto the Agilent Human miRNA V3 8X15K microarray (Agilent Technologies). The slides were scanned using a G2565BA scanner (Agilent). The data were extracted using Agilent Feature Extraction Software and analyzed by Nexus Expression Revision 2.0 (Biodiscovery). MiRs identified by microarray were validated by quantitative real time PCR. The potential targets of miR were identified by in-silico prediction algorithms using the Sanger miRBase (http://www.mirbase.org).

Results: A total of 11 miRs (miR-26a, -30a, -30e, -34a, 92a, -130a, -210, -1246, -1290, let7b and let7c) were differentially expressed in MSI-high tumors by microarray. Six of these miRNAs (let-7b, miR-210, -26a, -30e, -92a, and -130a) were validated by TaqMan-based stem-loop qPCR. Among them, miR-210, which has been reported to down regulate DNA homology-dependent repair (HDR) pathways through RAD52 gene, was upregulated in MSI ECs. Two additional potential targets involved in DNA damage repair system, $A L K B H 3$ (alkylation repair homolog 3, in E. coli) and DDI2 (DNA-damage inducible 1 homolog 2), were identified and confirmed by in vivo assays. An inverse correlation between the expression of miR-210 and expression of $A L K B H 3$ or DDI2 mRNA was observed in EC samples.

Conclusions: MicroRNAs are differentially expressed in MSI-high ECs. Increased expression of miR-210 may contribute to the pathogenesis of MSI-high ECs by downregulating genes that are involved in DNA damage repair.

1891 Integrated Genome Sequence, Epigenome, and Transcriptome Map of Bladder Cancer Development from Field Effects.

TMajewski, W Chung, Y Wu, DE Cogdell, J Bondaruk, S Zhang, SK Lee, SLee, L Shen, C Wei, SY Yoo, W Choi, D McConkey, JN Weinstein, KA Baggerly, J-P Issa, W Zhang, SE Scherer, BA Czerniak. UT MD Anderson Cancer Center, Houston, TX; Baylor College of Medicine, Houston, TX.

Background: Genomic maps that track the development of human cancer from its in situ precursor conditions to invasive disease can facilitate the search for genes and genomic sequences involved in the development of cancer. The identification of those chromosomal regions and their positional target genes as well as non-coding sequences that provide growth advantage for the incipient preneoplastic conditions can provide novel insights on molecular mechanisms of cancer development. Such an approach is also critical for the identification of novel biomarkers as well as targets for preventive and therapeutic strategies.

Design: We report on the construction of a high resolution whole-organ histologic and genetic map of bladder cancer development that tracks its progression from in situ precursor conditions to invasive clinically aggressive cancer using exome deep sequencing and 1M SNP-based Illumina genotyping combined with $\mathrm{CpG}$ island methylation and cDNA microarray technology platforms. This approach provided a high resolution integrated pattern of structural genome, epigenome, and transcriptome alterations that parallel the development of bladder cancer from early field effects to invasive disease.

Results: By analyzing genome-wide patterns of structural genomic (copy loss or gain), epigenomic, and transcriptome changes, we found three major waves of alterations associated with growth advantage of successive clones reflecting a stepwise conversion of normal urothelial cells into cancer cells. In particular, this approach is significant for the identification of specific chromosomal regions as well as epigenetically and transcriptionally modified gene ontologies involved in early clonal expansion of intraurothelial neoplasia and their clonal evolution to carcinoma in situ progressing to invasive cancer. These studies suggest involvement of ras/raf gene network in early clonal expansion of intraurothelial neoplasia.

Conclusions: This map represents a prerequisite for specific studies on the mechanisms of incipient phases of bladder cancer development and should have a significant impact on the understanding of human carcinogenesis.

1892 Integrated Signature of PI3K Pathway Activation in Prostate Cancer.

NE Martin, JA Sinnott, S Finn, G Fedele, MJ Stampfer, O Andren, S-O Andersson, L Mucci, M Loda. Dana Farber Cancer Institute \& Brigham and Women's Hospital, Boston, MA; Örebro University Hospital, Sweden; Harvard School of Public Health, Boston, MA.

Background: The PI3K pathway is commonly dysregulated in prostate cancer ( $\mathrm{PCa}$ ). Tumor heterogeneity and the complexity of the pathway have made it challenging to develop reliable signatures of PI3K pathway activation. We used an integrated approach of 4 immunohistochemical (IHC) markers and gene expression profiling (GEP) data to characterize activation of the pathway across more than 400 tumors.

Design: Men incidentally found to have PCa and enrolled in a watchful waiting cohort from Sweden (1977-1999) were used as the training cohort with a prostatectomy 
cohort of men from the Physicians' Health Study (1983-2004) used for validation. For each tumor, we determined IHC expression of PTEN, pAKT (Ser 473), pS6RP (Ser $240 / 244$ ), and stathmin on tissue microarrays using automated image analysis. We created a putative "PI3K score" based on the sum of the quartiles of abnormal staining for each marker. GEP for each sample was determined using the DASL platform for approximately 6100 genes.

Results: There were 304 and 99 men in the training and validation cohorts respectively. In pooled analyses, PTEN $(p=0.03)$, and stathmin $(p=0.005)$ IHC staining were significantly associated with the development of lethal disease. High PI3K scores were significantly associated with $\mathrm{Ki} 67$ in both training and validation sets $(\mathrm{p}<0.01)$. In the validation cohort, high PI3K scores were borderline significantly associated with increasing Gleason and death or metastatic disease ( $\mathrm{p}=0.05$ for both). Using prediction analysis of microarrays, 48 genes were identified in the training set which could significantly distinguish between low and high PI3K scores. When applied to the validation set, the area under the ROC curve was $74 \%$ (54-91\%) for correct classification of the two states. Gene set enrichment analysis identified a prior in vivo signature of pathway activation as being significantly enriched in the genes segregating high and low PI3K score tumors.

Conclusions: The combined staining pattern of PTEN, pAKT, pS6RP and stathmin appears to be significantly related to proliferation and clinical features of PCa. GEP data supports the hypotheses that these markers capture activation of the PI3K pathway. A signature of PI3K pathway activation could be used in clinical trials investigating agents targeting pathway components.

\section{Bone Marrow Aspirate Paraffin Blocks as Reliable Source for} Long-Term Protein Preservation.

M Nassiri, S Ramos, A Morales. Indiana University School of Medicine, Indianapolis; Univerity of Miami, FL.

Background: Currently there is no economical method available to preserve bone marrow samples for molecular studies. We have studied feasibility of preserving biomolecule including proteins in bone marrow aspirate particle preparation using a novel molecular friendly tissue processing system.

Design: Bone marrow aspirates particle preparations were fixed in Molecular Fixative ${ }^{\mathrm{TM}}$ and processed using Tissue-Tek ${ }^{\circledR}$ Xpress $^{\mathrm{TM}}$ (Sakura Finetek, Torrance, CA). Paraffin blocks were kept at room temperature for at least 18 months. Proteins were extracted from the freshly isolated samples, 50 micron thick sections of the paraffin blocks immediately after processing and after 18 months from the same block kept at room temperature. Commercial kits (Bio-Rad and Calbiochem) were used to isolate various subcellular compartments (nuclear, cytoplasmic organelles, cytoplasmic membrane) from the paraffin-embedded tissue. Isolated extracts were further separated on a fluid phase isoelectric focusing chamber (Bio-Rad Micro-Rotofor) and run on SELDI-TOF mass spectrometry on different protein-chip chemical surfaces. 2D gel electrophoresis with further spot excision and trypsin digestion was used to identify proteins using SEND ID chips. Immunohistochemistry was performed on paraffin-embedded tissue section for 20 major hematopoietic proteins.

Results: Protein profiling using mass spectrometry from paraffin embedded samples demonstrated similar pattern to fresh samples. Similar 2D gel pattern were obtained after 18 month interval from samples kept at room temperature. Immunohistochemistry was successful for all studied proteins.

Conclusions: It is feasible to use a novel formalin-free bone marrow particle preparation for preservation of high molecular weight macromolecules. Profiling using SELDI-TOF and specific protein identification were reproducible and similar after 18 month. This approach to preservation of bone marrow aspirate particles can serve as a cost-effective method for bio-repositories in clinical settings. Furthermore, preservation of bone marrow micro-architecture opens door for in situ molecular studies.

1894 Genome-Wide Massively Parallel Sequencing Using SOLiD ${ }^{\text {TM }} 4$ of Formalin Fixed Paraffin Embedded Prostate Cancer Tissue.

P Nikolov, R Menon, M Brown, V Scheble, F Fend, D Boehm, S Biskup, S Perner University Hospital Bonn, Germany; University Hospital Tuebingen, Germany; CeGaT $\mathrm{GmbH}$, Tuebingen, Germany; University of Tuebingen Medical Center II, Germany. Background: Alternation in the exome is considered to be one of the instigators of cancer. The exome forms $1.5 \%$ of the human genome, consisting of the genetic blueprint for protein synthesis and other functional gene products. SOLiD-Sequencing by Oligonucleotide Ligation and Detection (Life Technologies) has an accuracy of 99.94\%. This robust technique has proven to be helpful in detecting novel genes associated with prostate cancer genomic and chromosomal rearrangements, copy number variation, and nucleotide substitutions. The aim of this study was to compare whole exome sequencing of paired frozen/formalin fixed paraffin embedded (FFPE) tumor tissue with non-tumor FFPE tissue obtained from the same patient in order to detect genes involved in cancer progression.

Design: So far, we investigated one prostate sample. This sample was divided into FFPE tumor, fresh frozen tumor and FFPE non-tumor material, all derived from the same patient. DNA was isolated and purified from all three samples. These samples were then processed for library construction to carry out deep sequencing on the SOLiD 4 system.

Results: The high mapping stringency approach generated reliable values for the unique placed reads. Our results show highly similar ontarget reads within $+/-150$ bp for each sample, including $81.91 \%$ for the FFPE non tumor, $78.47 \%$ for FFPE tumor and $82.44 \%$ for frozen tumor. We detectably captured $98.19 \%$ of targeted exons for FFPE non-tumor, compared to the $98.32 \%$ for FFPE tumor and $98.52 \%$ for the frozen tumor samples. Using QV-filtering, we mapped 20560 SNPs to regions of interest, for each dataset. By comparing the SNP profiles between tumor and non- tumor sets, 1297 SNPs were found to be highly specific for the tumor samples. Out of these, we could identify 341 non-synonymous SNPs and 289 synonymous SNPs that will be further analyzed. Conclusions: This is the first study showing comparable exome sequencing results between the FFPE and corresponding frozen cancer tissues using SOLiD 4. Based on the promising results, the study will be continued on a much larger cohort exhibiting both localized and metastatized prostate cancer. The usage of FFPE blocks will open up the Pathology archives for exome-related studies that provide insight into the $\mathrm{PCa}$ genome, resulting in novel biomarkers and therapeutic targets discovery.

1895 Pathogen Discovery in Hematological Neoplasms and Inflammatory Diseases by High Throughput Sequencing of Human Tissues.

AI Ojesina, A Kostic, J Jung, C Pedamallu, G Getz, J Maciejewski, M Shipp, E Cesarman, J Aster, M Meyerson. Dana-Farber Cancer Institute, Boston, MA; Broad Institute of MIT anad Harvard, Cambridge, MA; Harvard Medical School, Boston, MA; Cleveland Clinic, OH; Weill Cornell Medical College, New York, NY; Brigham and Women's Hospital, Boston.

Background: Many diseases are thought to be caused by pathogens. However, many of these pathogens are unknown, possibly because they are present at very low frequencies in the diseased tissues. The advent of high throughput sequencing provides a unique opportunity to address this need. Our work is based on the premise that tissues from pathogen-driven diseases should contain both human and pathogenic nucleic acids. Therefore, high throughput sequencing, followed by computational subtraction of human sequences should result in the enrichment of candidate pathogenic sequences.

Design: We generated cDNA libraries from primary tissues across several hematological cancers and inflammatory diseases. These libraries were subjected to high throughput Illumina sequencing to generate $30-60$ million $76 \mathrm{bp}$ paired-end sequence reads per sample. Quality filtered reads were analyzed using our automated pipeline, PathSeq, which carries out several subtraction steps involving alignments to i) human genome sequence databases; ii) human transcriptome sequence databases; and iii) other vertebrate sequence databases. Residual sequence reads were then compared with microbial databases, either individually or as part of de novo assembled contigs.

Results: Using both frozen and formalin-fixed paraffin-embedded (FFPE) tissues, we have identified unique pathogenic sequences previously unassociated with hematological cancers and inflammatory diseases. In addition, our pathogen discovery pipeline works with both transcriptome and whole genome sequencing (WGS) data, and it is applicable to data across all high throughput sequencing platforms. Most notably, we are able to detect as low as 1 viral sequence per billion total sequences for WGS data, a sign of the sensitivity of our method. Furthermore, we are implementing a Cloud Computing version of the pipeline for public use by the general scientific community.

Conclusions: We have developed an integrated pipeline, PathSeq, for pathogen discovery in both frozen and FFPE tissues using a high throughput sequencingbased computational subtraction process. This presentation will include highlights of our pipeline and the results of our PathSeq analyses in hematological cancers and inflammatory diseases.

1896 The Human Protein Atlas.

F Ponten. Rudbeck Laboratory, Uppsala University, Sweden.

Background: In 2005, the first version of the Human Protein Atlas (www.proteinatlas. org) was released to provide a map of protein expression patterns. The underlying effort is an antibody-based proteomics strategy, applied to combine high-throughput generation of mono-specific antibodies with protein profiling on tissue and cell microarrays containing normal human tissues (144 individuals), cancer tissues (216 patients) and 50 cell lines. Additionally, subcellular protein expression patterns are determined in 3 human cancer cell lines using immunofluorescence.

Design: The current database includes protein profiling data and high-resolution images for over 11.000 antibodies. Each histology image has been manually annotated by a certified pathologist to provide a knowledge base for functional studies and to allow searches for protein profiles in normal and cancer tissue. The subcellular location for each protein target is reported based on manual annotation of immunofluorescent signals in analyzed cells. In November 2010 a new version 7 will be released to include knowledge-based protein expression data and images representing 10.000 unique proteins corresponding to $50 \%$ of the human genome.

Results: The systematic exploration of the human proteome has provided a new insight into global protein expression patterns and also created opportunities to identify various types of biomarkers with in silico based methods. To examine protein expression levels in tumors from larger patient cohorts specially designed tissue microarrays including clinical data associated with the corresponding patients are generated and immunostained with antibodies against selected targets, to allow for clinical validation of new cancer biomarkers.

Conclusions: The Human Protein Atlas represents a resource containing protein expression data with high-resolution images of human normal and cancer cells. Thi resource can be used for further analyses of cell type-specific expression patterns, including searches for potential cancer biomarkers. The mission is to create a map of the expression and localization of a majority of all human proteins, in both normal and cancer tissues, within a time frame of 5 years. References Uhlen M. \& Ponten F. Antibody-based proteomics for human tissue profiling. Mol Cell Proteomics 4, 384-93. (2005) Berglund L. et al. A gene-centric human protein atlas for expression profiles based on antibodies. Mol Cell Proteomics 7, 2019-27 (2008). Ponten F, Jirstrom K and Uhlen M. A Human Protein Atlas: A Tool For Pathology. J Pathology. 216, 387-93 (2008). Ponten F. et al. A global view of protein expression in human cells, tissues and organs. Mol Sys Biol. 5: 337 (2009) 
1897 Tissue Metablomics by High-Resolution ${ }^{1} \mathrm{H}$ NMR Spectroscopy: Discovery of Colon Cancer-Specific Metabolomic Biomarkers.

MH Roehrl, SS Wang, JY Wang. Boston Medical Center, MA; Brigham and Women's Hospital, Harvard Medical School, Boston, MA.

Background: Colorectal cancer remains among the top causes of cancer mortality. Metabolomics, the comprehensive global characterization of small molecules involved in cellular biochemical transformations, is a powerful epigenetic approach to understanding the functional pathobiology of cancer and its underlying metabolic aberrations. Highresolution liquid proton $\left({ }^{1} \mathrm{H}\right)$ nuclear magnetic resonance (NMR) spectroscopy is a sophisticated biophysical technique that allows a global, sensitive, and non-destructive interrogation of metabolomes extracted from human tissues.

Design: 40 paired samples of fresh cancerous and adjacent normal colonic tissues were chemically extracted by a methanol-chloroform-water mixture. Tissue metablomes were partitioned into aqueous and organic phases and subsequently examined by high-resolution ${ }^{1} \mathrm{H}$ NMR. Fourier-transformed spectra were binned, normalized, and analyzed for statistically significant differences between normal and cancer groups. Principal component analysis (PCA) and partial least squares (PLS) discriminant analysis were used to distinguish normal spectra from cancer and to develop cancer biomarker models.

Results: PCA and PLS discriminant analysis highly successfully distinguished normal spectra from cancer. Studies of organic spectra indicated that cholesterol, cholesterol esters, and phospholipids were elevated in cancer samples, while triacylglycerol levels were depressed. Poly-unsaturated lipids also appeared predominantly in tumor biopsies, whereas mono-unsaturated lipids were more common in normal colon tissue. Analysis of aqueous samples showed increased quantities of uridine diphosphate $\mathrm{N}$-acetyl-D-glucosamine, uracil, proline, lactate, O-phosphoethanolamine, glutamate, aspartate, oxidized glutathione, inosine monophosphate, and taurine in cancer spectra. In contrast, the metabolites glucose, myo-inositol, creatine, and scyllo-inositol were more prominent in normal spectra.

Conclusions: We discovered quantiative and qualitative metabolite biomarkers of human colorectal cancer. Using bioinformatics to map metabolomic differences onto network models of cellular metabolism, intriguing connections were discovered that hint at concerted large-scale metabolic readjustments in tumors and that provide exciting new directions for diagnostic test and drug target development. We show that metabolomics is a powerful and promising novel experimental approach and adds to the the toolbox of systems pathology of disease.

1898 Proteomic Profiling of Pancreatic Cancer: Validation of a Neuronal Signature.

D Rundell, C Thompson, S Vareed, VB Bhat, V Vasu, M Shabahang, J McLoughlin, A Sreekumar, A Rao. Scott \& White Hospital, Temple, TX; Medical College of Georgia, Augusta; Agilent Technologies, Wilmington, DE

Background: Pancreatic ductal adenocarcinoma (PDAC) is the fourth leading cause of cancer death in the United States with a 5 year survival rate of $4 \%$. Biomarkers that are detectable early in the course of disease have the potential to markedly improve clinical outcomes.

Design: We profiled the secretory proteome of pancreatic duct fluid in 25 samples (18 carcinomas and 7 benign) using a MudPIT approach with HPLC-Chip nano ESIMS/MS analysis. A total of 433 proteins were detected of which 56 were found to be significantly elevated in PDAC. Alpha Synuclein (SNCA) was in this differential set and was further investigated as potential marker.

SNCA expression was correlated to tissue expression by immunoblot and immunohistochemistry. Tissue microarrays (TMA) from 76 PDAC, 9 pancreas mucinous cystadenomas, 17 intraductal papillary mucinous neoplasms (IPMN), 6 intraductal papillary mucinous carcinomas (IPM) with and without invasion, 45 benign pancreatitis cases and 44 carcinomas from other sites were examined. SNCA staining was scored as the sum of the intensity $(0-3+)$ and extent $(0-4+)$. Positive staining was defined to be a total score of 5 or greater. Additionally tumors were stained with HMB-45 and FontanaMasson to investigate neurological differentiation in SNCA positive PDAC.

Results: Immunoblot analysis confirmed elevated levels of SNCA in tissues from pancreatic adenocarcinoma patients compared to controls. Weak to no staining was noted for SNCA in a vast majority $(\sim 95 \%)$ of the pancreatitis specimens $(n=28)$ while approximately $83 \%$ of the PDAC specimens $(n=76)$ exhibited a positive staining pattern. No neuronal differentiation was identified in the SNCA positive cases. There was positive staining in 13 of the IPMN and IPM carcinomas and all of the mucinous cystic neoplasms. Positive staining was noted in a majority of the colon (5 of 6), gastric ( 5 of 6 ) and lung adenocarcinomas ( 6 of 6 ). No staining was noted in hepatocellular and urothelial carcinomas and only a minority of breast ductal carcinomas (2 of 6) showed positive staining for SNCA

Conclusions: Synuclein A is overexpressed in pancreatic duct fluid and tissues derived from patients with pancreatic adenocarcinoma and pancreatic mucinous tumors indicating that this protein may be involved in the dysregulated growth of pancreatic neoplasms. Mechanistic understanding the role of SNCA in PDAC, GI and lung adenocarcinomas may reveal prognostically relevant pathways.

1899 Expression Profiling of Holoclone and Non-Holoclone Derived Cell Lineage in Prostate Cancer.

YM Salley, MF Gallagher, PC Smyth, CM Martin, OM Sheils, JJ O'Leary. University of Dublin, Trinity College, Dublin, Ireland.

Background: Prostate cancer is a heterogeneous disease and is the second most common cause of cancer resulting in male deaths caused by cancer. Stem-like cells have been identified in several malignancies including prostate cancer and are thought to drive primary tumorigenesis through self-renewal and differentiation. Additionally, persistence of stem cells post-therapeutic intervention has been proposed as an explanation for metastasis and recurrence. Holoclones are a tightly packed clone of smal cells generally thought to contain stem cells and progenitors. The aim of this study was to generate an expression profile of holoclone derived cell lineage in prostate cancer. Design: In this study, holoclones were cultured using a high salt-soft agar assay for LNCaP (metastatic carcinoma) and PC-3 (non-metastatic adenocarcinoma) cell lines. Microarray analysis was carried out on cell lines and holoclone derived cells $(n=4)$ and analysed by XRAY software using the following criteria; FDR $\leq 0.05$ and FC \pm 2. Gene ontology of biological processes and pathways was generated with DAVID and PANTHER. Genes were selected and validated using a quantitative Real Time TaqMan ${ }^{\circledR}$ PCR method

Results: Holoclones were generated from cell lines (LNCaP, PC-3) using a high salt-soft agar assay. PC-3 versus PC-3 holoclones demonstrated 228 significant differentially regulated genes and LNCap versus $\mathrm{LNCaP}$ holoclones demonstrated 44 significant differentially regulated genes. Both PC-3 and $\mathrm{LNCaP}$ holoclones displayed a unique stemness expression signature.

Conclusions: Microarray analysis demonstrated that holoclone derived cells display significant stemness gene expression when compared to the founder cell lines. Analysis also indicated different stemness characteristics between PC-3 and LNCaP holoclones. Future work will consist of further characterisation of holoclone cells. Acknowledgements: Prostate Cancer Research Consortium.

1900 Recurrent Cytogenetic Alterations in Radiation Induced (RI) Abdominal Malignant Mesothelioma (AMM)

AN Sireci, B Levy, TRobert, JL Chen, NOdelia, BCAlain. Columbia University Medical Center, New York, NY.

Background: Abdominal malignant mesothelioma (AMM) is a rare tumor of the peritoneum that has been linked to asbestos exposure and past radiation therapy to the abdomen. Other than patient history, there is no established method to differentiate the two etiologies. We describe recurrent copy number alterations that differ between RI tumors and other AMM.

Design: Needle-dissected tumor from 7 RI AMM tumors (cases) and 34 non-RI tumors (controls) were studied for copy number $(\mathrm{CN}$ ) alteration (Affymetrix SNParray 6.0). CN data were analyzed using NEXUS software. A list of $\mathrm{CN}$ alterations that significantly predicted radiation exposure at $\mathrm{p}<0.025$ was compiled. All tumors were of epithelioid morphology.

Results: Loci of $\mathrm{CN}$ alterations associated with tumor etiology were identified. Potential genes of interest within these loci include those involved in cell signaling, cell cycle regulation, cellular cytoskeleton, transcription/translation modification, metabolism, protein ubiquitination and cell stress response.

Representative chromosomal regions involved in CN alterations in RI-AMM

\begin{tabular}{|l|l|l|l||}
\hline Chromosome & Alteration & Distributions & No. of Genes Involved \\
\hline $1 \mathrm{p}$ & Loss & $3 / 7 \mathrm{RI}, 3 / 34 \mathrm{ctrl}$ & 10 \\
\hline $3 \mathrm{p}$ & Loss & $3 / 7 \mathrm{RI}, 8 / 34 \mathrm{ctrl}$ & 3 \\
\hline $3 \mathrm{p}$ & Loss & $4 / 7 \mathrm{RI}, 2 / 34 \mathrm{ctrl}$ & 8 \\
\hline $3 \mathrm{q}$ & Loss & $5 / 7 \mathrm{RI}, 9 / 34 \mathrm{ctrl}$ & 1 \\
\hline $3 \mathrm{q}$ & Loss & $2 / 7 \mathrm{RI}, 0 \mathrm{ctrl}$ & 15 \\
\hline $3 \mathrm{q}$ & Gain & $2 / 7 \mathrm{RI}, 0 \mathrm{ctrl}$ & 4 \\
\hline $3 \mathrm{q}$ & Loss; Gain & $4 / 7 \mathrm{RI}, 2 / 34 \mathrm{ctrl} ; 3 / 7 \mathrm{RI}, 1 / 34 \mathrm{ctrl}$ & 1 \\
\hline $3 \mathrm{q}$ & Gain & $2 / 7 \mathrm{RI}, 0 / 34 \mathrm{ctrl}$ & 9 \\
\hline $15 \mathrm{q}$ & Loss; Gain & $3 / 7 \mathrm{RI}, 2 / 34 \mathrm{ctrl} ; 0 / 7 \mathrm{RI}, 9 / 34 \mathrm{ctrl}$ & 1 \\
\hline
\end{tabular}

Conclusions: We have identified recurrent copy number alterations in RI AMM that differentiate them from control cases of AMM. These genetic changes may be helpful in diagnosing AMM and in determining mechanism of oncogenesis.

1901 microRNA Profiling in Lung Cancer Diagnosis and Staging.

CC Solomides, B Evans, P Li, V Kumar, $R$ Vadigepalli, SC Peiper, Z Wang. Thomas Jefferson University, Philadelphia, PA.

Background: Carcinoma of the lung is the leading cause of death among cancer worldwide. With the advent of therapies that have selective efficacy between squamous cell carcinoma (SCC) and adenocarcinoma (AC), the distinction between these two types is critical. Although immunohistochemical markers are used, there are still cases difficult to classify. Different cell types have distinct microRNA (miRNA) expression profiles. Since miRNA can be efficiently extracted from formalin-fixed, paraffin-embedded (FFPE) tissues, we determine the ability of miRNA profiling to distinguish SCC from $\mathrm{AC}$ and to classify poorly differentiated variants.

Design: De-identified cases $(\mathrm{n}=12)$ were selected from the surgical pathology archive. FFPE blocks for analysis included AC $(n=5)$, SCC $(n=4)$, normal lung from areas adjacent to tumors $(n=4)$, small cell carcinoma $(n=1)$, and two poorly differentiated specimens. Total RNA was extracted from FFPE using Recover All total nucleic acid extraction kit (Ambion). RNA was labeled and hybridized to the microarray according to the manufacturer's procedure. The median of the $\log 2$ transformed raw signal intensity data was processed through an invariant normalization workflow to identify "invariant" miRNAs that have similar expression across the samples and "hypervariables" with expression that varies significantly across the samples. The "invariant" miRNA were used to normalize data across all of the samples using quantile normalization and this yielded 34 microRNAs that are differentially expressed.

Results: A panel of 4 miRNAs was identified for differentiating SCC from AC. Expression of miR2 25 was dramatically increased and miR100, miR150, and miR26b expression was reduced only in SCC but not in AC. Another panel of 16 miRNAs that differentially expressed in normal or cancer specimens were identified Thirteen were significantly down-regulated in lung cancers compared to the corresponding normal lung tissues ( $\mathrm{p}$ value: 0.0004 0.058). Expression of 3 miRNAs (miR21, miR210 and 
miR224) were 3-5-fold increased in the tumor tissues compared to normal lung tissues ( $p$ value: $0.0001 \sim 0.1$ ). The altered gene expression profiles correlated with tumor stage independent of cell type.

Conclusions: We identified a panel of miRNAs that are differentially expressed in SCC and $\mathrm{AC}$ and another independent panel of miRNAs that correlates with cancer stage. Both panels will be further validated with large cohorts of tumor samples using realtime PCR. This approach shows significant potential for clinical utility in the diagnosis and prognosis of lung cancer.

1902 BRCA1 Functions as a 5'- Nuclease Via PIN Domain Identified by Multiple Protein Sequence Alignments.

$S$ Tatishchev, E Spiteri, J Lopategui. Cedars-Sinai Medical Center, Los Angeles, CA. Background: BRCA1 has been implicated in a multitude of biological processes. It co-localizes via conserved RING \& BRCT domains with a number of proteins involved in DNA damage repair and cell cycle control. Despite extensive research, BRCA1 function has remained elusive. Thus, identification of new conserved domains is paramount to uncovering BRCA1 biological function. Recently, crystallographic studies and bioinformatics analyses of orthologous proteins have revealed a number of highly conserved amino acids up to hundreds of residues apart that form functional domains only when put in close proximity to each other within a tertiary protein structure. Using sequence alignment software and domain database search we tested our hypothesis that multiple alignments between BRCA1 proteins would reveal a set of phylogenetically conserved amino acids capable of forming a functional domain at the level of BRCA1 tertiary structure.

Design: BRCA1 protein sequences from divergent mammalian taxa were identified in the NCBI database. The sequences were aligned using the free online software ClustalW2. ExPASy Proteomics Server and Pfam protein database were used to identify a functional domain.

Results: An analysis of BRCA1 sequence alignments identified 5 highly conserved amino acids across all mammalian orders (Fig 1).

\section{censs rovours}

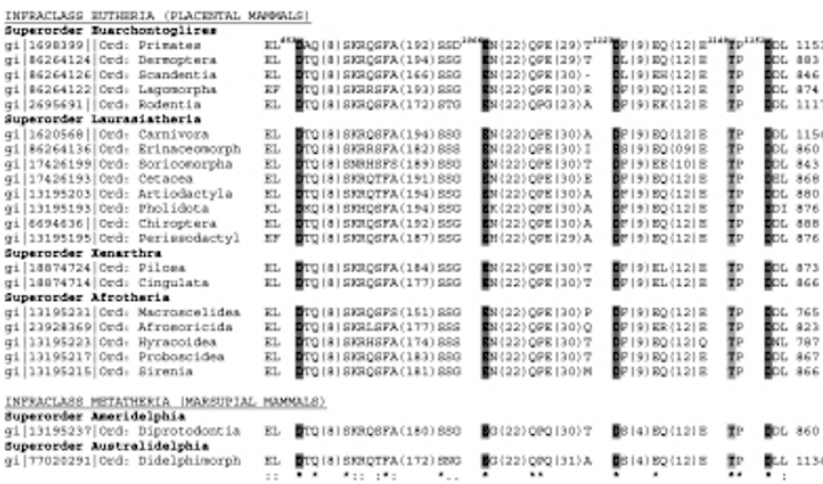

Four acidic residues (D853, E1066, D1123, D1151) and threonine (T1149) within exon 11 were identified as the PIN domain sequence $\left.{ }^{\mathrm{N}-\text { term }}-\mathrm{D}-\ldots-\mathrm{D} / \mathrm{E}-\ldots . \mathrm{D}-\ldots-\mathrm{T} / \mathrm{S} x \mathrm{D} \mathrm{C}^{\mathrm{C}-\mathrm{term}}\right)$. In PIN domains the acidic residues are spatially clustered to support coordination of $\mathrm{Mg}^{2+}$ ions forming the active site of 5'-nucleases. In eukaryotes, PIN-domain containing proteins are an integral part of the nonsense-mediated decay (NMD) process that functions to eliminate transcripts with premature termination codons.

Conclusions: Discovery of the PIN domain within BRCA1 sequence, for the first time, provides an insight into its potential biological function as a 5 '-nuclease involved in tumor suppression \& possibly NMD. In addition, the presented strategy to functionally characterize proteins involved in oncogenesis, such as BRCA1, is straightforward, requiring only publicly available protein sequences spanning divergent taxonomic groups and a handful of user-friendly bioinformatics tools.

\section{Characterization of Markers for Pancreatic Cancer Obtained by} Proteomic Profiling.

C Thompson, D Rundell, S Vareed, V Bhat, V Vasu, M Shabahang, J McLoughlin, A Sreekumar, A Rao. Scott \& White Hospital, Temple, TX; Medical College of Georgia, Augusta; Agilent Technologies, Wilmington, DE

Background: Pancreatic ductal adenocarcinoma (PDAC) is the fourth leading cause of cancer death in the United States. Currently, there are no clinically useful biomarkers for the detection of clinically occult PDAC. We are using a proteomics-based approach to identify possible PDAC biomarkers.

Design: 25 samples of pancreatic duct fluid (18 carcinomas, 7 benign) were analyzed using label-free mass spectrometry coupled to SDS-PAGE and Cation-Exchange Chromatography. 433 proteins were detected of which 56, including Purine Nucleoside Phosphorylase (NP), were found to be significantly elevated in PDAC. The expression of NP was then correlated to tissue expression by immunoblot and immunohistochemistry (IHC). Tissue microarrays (TMA) comprised of 76 PDAC, 9 pancreas mucinous cystadenomas, 12 intraductal papillary mucinous neoplasms (IPMN), 6 intraductal papillary mucinous carcinomas with and without invasion, 45 benign pancreatitis samples, and 44 carcinomas from non-pancreatic sites were examined. NP staining was scored for extent $(0-4+)$ and intensity $(0-3+)$ with a total staining score defined as the sum of the intensity and extent scores. Positive staining was defined as a total score $>4$. Results: Immunoblot analysis for NP in serum revealed two bands at $\sim 35 \mathrm{KDa}$, with a unique upper band seen in PDAC tissue and sera that was low to undetectable in benign cases. 61 of 76 PDAC cases were positive for NP by IHC. NP was predominantly negative (average total staining score $=1$ ) in cases of IPMN, IPM carcinomas, mucinous cystadenomas and pancreatitis. Weak NP staining (average total score =3) was noted in gastric and colonic adenocarcinomas. Adenocarcinomas from the breast and lung as well as hepatocellular and urothelial carcinomas were essentially negative.

Conclusions: NP is overexpressed in pancreatic duct fluid and tissues derived from patients with pancreatic adenocarcinoma indicating that this protein may be involved in the pathogenesis of PDAC. NP, the rate limiting enzyme in the purine salvage pathway, is operational during inflammation as well as neoplastic progression. This study highlights $\mathrm{NP}$ as a possible unique marker linking the pathogenesis of PDAC with antecedent pancreatitis. The absence of significant NP staining in pancreatic mucinous tumors may indicate different mechanistic pathways in these neoplasms.

\section{A Personalized Medicine Approach to Lung Cancer and} Melanoma.

C Vnencak-Jones, Z Su, L Fohn, C Coffin, A Gonzalez, D Itani, C Shi, J Sosman, $W$ $P a o$. Vanderbilt University Medical Center, Nashville, TN.

Background: Collectively $~ 300,000$ new cases of lung cancer and melanoma will be diagnosed in the US in 2010. Long term survival for patients with metastatic disease is poor. Therapy targeted against aberrant tumor proteins has demonstrated promising results, but only subsets of tumors harbor such aberrations. To facilitate personalized cancer medicine, we developed and implemented clinical DNA-based molecular profiling on all non-squamous non-small cell lung cancer and melanoma patients treated at our institution. This approach identifies patient specific tumor mutations, enables personalized targeted therapy and intends to optimize treatment and improve outcome.

Design: A multidisciplinary team developed and implemented this process. Lung and melanoma specific DNA-based assays using the SNaPshot multiplex kit (Applied Biosystems) and fluorescent PCR coupled with fragment analysis were designed to detect common somatic mutations linked with outcomes to existing or emerging targeted therapies. Each assay was vigorously tested and validated within a research lab prior to transition into the clinical lab and the performance and sensitivity of each assay was independently verified in the clinical lab. Implementation of the initiative involved: establishment of a detailed process of tissue submission; education of clinicians, pathologists and laboratory staff and development of an effective reporting system within the electronic medical record.

Results: The lung cancer panel includes analysis of $\sim 40$ mutations (including indels) in 9 genes: EGFR, HER2, KRAS, BRAF, PIK3CA, MEK, NRAS, AKT1 and PTEN. The melanoma panel detects 43 mutations in 6 genes: BRAF, KIT, NRAS, GNAQ, GNAII, and $C T N N B 1$. Sensitivity of the assays is $5 \%$, lab turn around times is 8 days and $3 \%$ of samples have been rejected for insufficient material or gave no results. Mutation frequencies are similar to that quoted in the literature, and results have been used to guide treatment options. Logistical challenges have included: delays in tissue block acquisition from outside facilities; improper submission of specimens; incomplete reflex testing for all specimens and insufficient staffing and equipment.

Conclusions: In $<1$ year, a collaborative and multidisciplinary team launched a personalized cancer medicine initiative in which lung cancers and melanomas are routinely profiled for disease-specific mutations linked to targeted therapies. This practice can now be expanded to other disease processes to facilitate adoption of genetically-informed medicine to improve clinical outcome.

1905 Aldehyde Dehydrogenase (ALDH) Activity Segregates Murine Pancreatic Cancer Stem Cells into Distinct Phenotypic Subtypes Which Dictate Histopathologic Tumor Grade.

DA Winer, SE Seeley, WW Tseng, JM Zahn, MN Alonso, S Winer, H Leong, M Kvezereli, $H J$ J, AM Lowy, EG Engleman. Stanford University, Palo Alto, CA; University Health Network, Toronto, ON, Canada; Moores Cancer Center, University of California at San Diego.

Background: Recent evidence suggests that the growth of tumors is driven by distinct cells with the ability to self renew and differentiate, called cancer stem cells (CSCs). Phenotypic diversity within the cancer stem cell (CSC) pool may account for the emergence of distinct patterns of tumor progression and drug resistance. However, because robust models for studying such diversity are lacking, fundamental aspects of CSC biology remain unknown.

Design: Cancer stem cells were isolated directly from pancreatic tumors and from three cell lines (primary tumor and liver metastases) derived from the commonly studied LSL$\mathrm{Kras}^{\mathrm{G} 12 \mathrm{D} /+}$; LSL-p53 ${ }^{\mathrm{R} 172 \mathrm{H} /+}$; Pdx-1-Cre mouse model of pancreatic cancer. These cells were analyzed using a comprehensive stem cell surface marker and genomic mRNA expression profiling approach and then partitioned according to ALDH functional activity using the Aldefluor assay. The resulting subsets of cells were studied in vitro and in vivo in the presence of an intact immune system for tumorigenic and stem cell properties.

Results: Epithelial cancer cells isolated from the LSL-Kras ${ }^{\mathrm{G} 12 \mathrm{D} /+} ; \mathrm{LSL}-\mathrm{p} 53^{\mathrm{R} 172 \mathrm{H} /+} ; \mathrm{Pdx}-$ 1-Cre mouse model of pancreatic cancer are highly enriched in CSCs. When these cells are partitioned according to ALDH activity levels, they yield poorly tumorigenic ALDH negative $\left(\mathrm{ALDH}^{\text {neg }}\right)$ cells and highly tumorigenic ALDH positive $\left(\mathrm{ALDH}^{\mathrm{pos}}\right)$ cells. ALDH ${ }^{\text {pos }}$ cells are further partitioned into $\mathrm{ALDH}^{\mathrm{dim}} \mathrm{CSCs}$, which give rise to high grade poorly-differentiated carcinomas that metastasize rapidly, and $\mathrm{ALDH}{ }^{\text {bright }} \mathrm{CSCs}$, which give rise to low grade well-differentiated glandular carcinomas with reduced metastatic potential. Relative to their $\mathrm{ALDH}^{\text {bright }}$ counterparts, $\mathrm{ALDH}^{\mathrm{dim}} \mathrm{CSC}$ exhibit morphological features and transcriptional alterations consistent with epithelial to mesenchymal transition (EMT).

Conclusions: These findings demonstrate the existence of multiple, pathology-defining pancreatic CSC subpopulations in a common mouse model, and provide highly tractable systems for their further characterization. 


\section{Techniques}

1906 Breast Tissue Imaging with Light Coherence Tomography: A Pilot Study.

O Assayag, A Burcheri, M Antoine, B Sigal, C Boccara. ESPCI ParisTech, France; Tenon Hospital, Paris, France; Curie Institute, Paris, France; LLTech, Paris, France.

Background: Breast cancer is the most common cancer and the second leading cause of death for women in western countries. Despite advances in early detection, breast imaging, and increased diagnosis of small tumors (cT1), up to $36 \%$ of breast-conserving surgery patients require subsequent excisions to achieve disease-free margins. The peroperatory evaluation of margins during surgery is time consuming, and the research for new techniques is justified. This pilot study aims to evaluate Light Coherence Tomography (Light-CT) as a tool for optical biopsies and imaging of benign and malignant human breast tissues.

Design: A Light CT scanner based on Full Field Optical Coherence Tomography (FFOCT) has been used to image human breast tissues. The light CT scanner has $\mathrm{a}<2 \mu \mathrm{m}$ axial resolution and $<2 \mu \mathrm{m}$ transverse resolution. 40 breast specimens have been imaged from at least 10 different patients covering healthy tissues from post and pre-menopausic women, lobular and ductal carcinoma, and sentinel nodes, after or without fixation. The time required and safety of a Light-CT imaging procedure have also been evaluated.

Results: Good structural correspondence is observed between Light-CT images and histological sections.

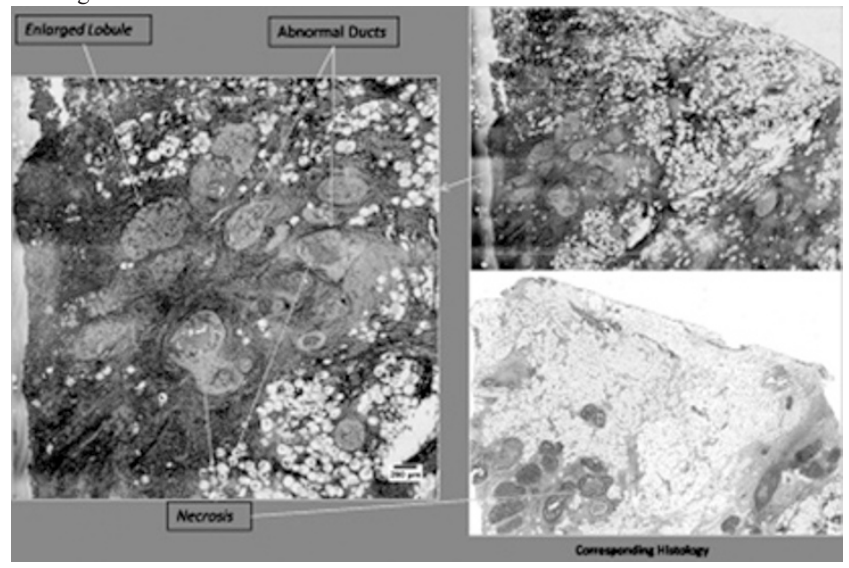

Characteristic features such as ducts, lobules, vessels, adipous, fibrous and necrotic tissue can be identified from Light-CT images and are recognized on normal and malignant tissue. Morphometric data such as the size of lobules and ducts and thickness of the parietal wall of the ducts and vessels as characteristics of these structures related to the presence of elastic tissue, can provide an additional diagnostic information. 9x9mm Light-CT images have been obtained in 2,5 minutes. Light-CT technology is safe since histology has been possible on $100 \%$ of the breast tissue samples.

Conclusions: We have demonstrated that the light CT technology is a fast and safe tool that can be used under clinical conditions. The Light CT images allow to recognize breast tissue structures and pathologies by comparison with traditional histology.

1907 Validation of Quantitative Real Time PCR for JAK2 Mutation Detection by Using $2^{-\Delta \mathrm{ct}}$ Method.

R Batra, C-K Huang, Q Pan. St Luke's Roosevelt Hospital Center, University Hospital of Columbia University College of Physicians and Surgeons, New York, NY; Montefiore Medical Center, Albert Einstein College of Medicine, New York, NY.

Background: The V617F mutation of the Janus kinase2 (JAK2) gene is a useful biomarker for the diagnosis of myeloproliferative disorders (MPD) such as polycythemia vera (PV), essential thrombocythemia (ET) and myeloid metaplasia with myelofibrosis (MF). JAK2 V617F is a gain of function mutation that leads to clonal proliferation; it is present in about $95 \%$ of PV cases and $50 \%$ of ET and MF cases. The aim of this study was to compare 2 methods for JAK2 mutation detection such as restriction fragment length polymorphism (RFLP) and real time PCR with $2^{-\Delta \Delta C t}$ calculation.

Design: In a retrospective cohort study, 254 samples were analyzed for genomic DNA from blood or bone marrow with the RFLP assay and real time PCR. In RFLP, PCR is used to amplify the portion of JAK2 mutation bearing region. Amplicon products are then digested with the Bsa XI restriction enzyme which generates products of 30bp, 97bp and 140bp from a $267 \mathrm{bp}$ amplicon. Samples harboring a V617F JAK2 mutation don't have the Bsa XI restriction enzyme leaving the $267 \mathrm{bp}$ amplicon intact. Capillary electrophoresis is used to resolve the different sized amplicon products. Real time PCR using a mutation specific primer pair detects the absolute quantity by determining the copy number of the genomic DNA by using the $2^{-\Delta \Delta C t}$ calculation, in this study. The PCR cycle in which a threshold signal is generated above the background noise is called the threshold cycle $(\mathrm{Ct})$. Positive result means that the $\mathrm{Ct}$ value of the tested sample is $\leq 40$

Results: Sensitivity of real time PCR is as low as $0.01 \%$ (1 positive cell per 10,000 cells) whereas that of RFLP is $5.0 \%$ ( 5 clonal cells in 100 normal cells). Out of 254 samples, 31 samples were positive with real time-PCR of which only 24 were positive with RFLP; that is $77.4 \%$ concordance between 2 methods. In the remaining 7 cases, the results were either normal or indeterminate on RFLP whereas the real time-PCT $2^{-\triangle \Delta C t}$ calculation in these subjects ranged from $0.02-0.4 \% .4$ cases with indeterminate results on RFLP were negative on real time-PCR.

Conclusions: This study suggests real time PCR as a more sensitive method for JAK2 mutation detection, which can also quantify the genomic DNA. The $2^{-\Delta \Delta \mathrm{Ct}}$ method reduces the time needed for preparation of standards and making a standard curve. In addition it reduces the contamination risk, reagent usage and the overall cost by reducing the number of reactions run each time.

1908 RNA Preservation in FFPE Tissues Requires Pre-Analytical Standardized Processing.

$G$ Bussolati. University of Turin, Italy.

Background: Preservation of RNA for Gene Expression profiling is notoriously poor in Formalin Fixed Paraffin Embedded (FFPE) tissues, thus hampering extended gene sequencing in routinely processed histo-pathological tissues.

Design: Our hypothesis was that RNA degradation in surgical biopsies is caused by RNAse activation before and during formalin fixation and that low temperature $\left(4^{\circ} \mathrm{C}\right)$ could inhibit this activity and prevent degradation. In our Hospital (a large, pavillon hospital in Piedmont, Italy) the traditional habit of transferring specimens from the surgical theatre to the Pathology laboratory while immersed in large boxes filled with formalin was substituted by Under-Vacuum sealing (UVS) using the TissueSAFE ${ }^{\mathrm{TM}}$ apparatus. Specimens, routinely U.V. sealed immediately after surgical removal, were kept at $4{ }^{\circ} \mathrm{C}$ for 1-72 h. until transfer. Grossing, fixation in Phosphate-buffered Formalin (PBF) and paraffin embedding followed. Selected specimens were processed by a 24 h. fixation in PBF at controlled temperature.

Results: UVS was well accepted by both Surgery and Pathology staff and this procedure, now in daily use since 2 years, assured proper histological and immunohistochemical quality as well as RNA preservation (RIN values above 7). Fixation in $\mathrm{PBF}$ at controlled conditions improved RNA preservation and gene expression analysis of paraffin-embedded tissues.

Conclusions: Standardization of pre-analytical processing is mandatory for a proper preservation and processing of surgical biopsies. Their transfer in UVS assured standard quality for structural, antigenic and nucleic acids analysis.

1909 Choosing an Immunohistochemistry (IHC) System? A Comparison of $\mathbf{3}$ IHC Stainers and Polymers from Different Manufacturers.

$Q$ Dai, ES Reisenbichler, MS Rasco, DK Horton, O Hameed. University of Alabama at Birmingham.

Background: IHC is routinely used in contemporary pathology practice and its quality depends upon many factors including IHC instruments and detection systems. The aim of this study was to compare the IHC results obtained using different open immunostainers and polymer detection kits.

Design: Different control sections for 9 antigens (CD3, CD20, CD45, CK, CK5/6, KI67, TTF1, BCL1, and S100) were sequentially evaluated by IHC utilizing 3 antibodies (Abs) from different sources [BIOCARE (BC), LABVISION (LV), DAKO (DK)], 3 different immunostainers (BC, LV, DK), and 3 different polymers (BC, LV, DK), generating 243 possible $\mathrm{Ab} / \mathrm{immunostainer/polymer} \mathrm{combinations.} \mathrm{The} \mathrm{intensity}(0-3+)$ and percentage of cells staining $(0-100 \%)$ were used to generate an $\mathrm{H}$-score $(0-300)$. Technical issues (loss of tissue, failure of staining, no counterstain) were recorded as absent, minor (still interpretable), or major (not interpretable), while background staining was recorded as present or absent.

Results: There was variation in the staining of the different antigens when utilizing the unique $\mathrm{Ab} / \mathrm{immunostainer/polymer} \mathrm{combinations} \mathrm{from} \mathrm{the} \mathrm{same} \mathrm{manufacturer}$ (figure 1), with different overall mean $\mathrm{H}$-scores for BC, LV, and DK (171, 199, and 218 , respectively; $\mathrm{P}=0.019$, paired ANOVA).

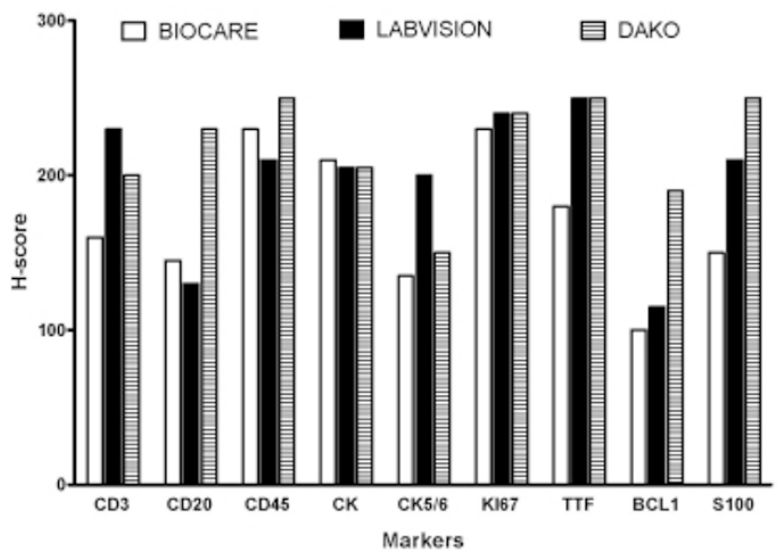

Comparison of staining between the 3 immunostainers (regardless of Ab/polymer) also showed different overall mean $\mathrm{H}$-scores $(175,199$, and 201 , respectively; $\mathrm{P}=0.036$, paired ANOVA), as did comparison between the 3 polymers (regardless of $\mathrm{Ab}$ / immunostainer) (194, 204 and 177, respectively; $\mathrm{P}=0.0003$, paired ANOVA). The overall incidence of technical problems across immunostainers was $18 \%, 23 \%$, and $20 \%$ for BC, $\mathrm{LV}$, and DK, respectively $\left(\mathrm{P}>0.05, \mathrm{X}^{2}\right)$ with major issues representing $43 \%, 26 \%$ and $19 \%$ of these issues, respectively $\left(\mathrm{P}>0.05, \mathrm{X}^{2}\right)$. Background staining was seen in $15 \%$, $14 \%$, and $9 \%$ of cases utilizing the $\mathrm{BC}, \mathrm{LV}$, and $\mathrm{DK}$ polymers $\left(\mathrm{P}>0.05, \mathrm{X}^{2}\right)$. 